\title{
Multi-agent Justification Logic: communication and evidence elimination
}

\section{Bryan Renne}

Received: 25 March 2010 / Accepted: 2 June 2011 / Published online: 5 July 2011

(C) The Author(s) 2011. This article is published with open access at Springerlink.com

\begin{abstract}
This paper presents a logic combining Dynamic Epistemic Logic, a framework for reasoning about multi-agent communication, with a new multi-agent version of Justification Logic, a framework for reasoning about evidence and justification. This novel combination incorporates a new kind of multi-agent evidence elimination that cleanly meshes with the multi-agent communications from Dynamic Epistemic Logic, resulting in a system for reasoning about multi-agent communication and evidence elimination for groups of interacting rational agents.
\end{abstract}

Keywords Justification Logic · Dynamic Epistemic Logic $\cdot$ Evidence elimination

\section{Introductory example}

Consider the following email exchange among friends planning a party.

$x_{1}\left\{\begin{array}{l}\text { To: Bob, Charlie From: Anne } \\ \text { If the cheese store is still open, then I'll bring the cheese tonight. }\end{array}\right.$

$x_{2}\left\{\begin{array}{l}\text { To: Anne, Bob From: Charlie Re: } x_{1} \\ \text { OK, Anne. If you bring the cheese, then I'll bring the crackers. }\end{array}\right.$

$x_{3}\left\{\begin{array}{l}\text { To: Bob, Charlie From: Anne } \\ \text { I just checked. The cheese store is still open! }\end{array}\right.$

$x_{4}\left\{\begin{array}{l}\text { To: Anne, Bob From: Charlie Re: } x_{3} \\ \text { It's great the store's still open! I'll go get the crackers. }\end{array}\right.$

After this sequence of messages, Bob and Charlie are each able to conclude that Anne is bringing the cheese. After all, each of Bob and Charlie has message $x_{1}$ as

\footnotetext{
B. Renne ( $\varangle)$

Faculty of Philosophy, University of Groningen, Groningen, The Netherlands e-mail: bryan@renne.org
} 
evidence that "Anne will bring the cheese if the store is open" along with message $x_{3}$ as evidence that the store is indeed open. So by combining their evidence $x_{1}$ and $x_{3}$, they each have evidence that Anne will bring the cheese.

Furthermore, since Charlie replied to each of Anne's messages $x_{1}$ and $x_{3}$ with his own messages $x_{2}$ and $x_{4}$, which indicates that Charlie has received and understood each of Anne's messages, Bob not only has his combined evidence $x_{1}$ and $x_{3}$ that Anne will bring the cheese but can also see on the basis of Charlie's messages $x_{2}$ and $x_{4}$ that Charlie too has combined evidence $x_{1}$ and $x_{3}$ to conclude that Anne will bring the cheese.

But now we add a twist to the story with the following private message from Anne to Bob.

\section{$x_{5}\left\{\begin{array}{l}\text { To: Bob From: Anne } \\ \text { Bob, I messed up! The store is closed! Charlie is going to flip! }\end{array}\right.$}

Clearly, this message has the effect of causing Bob to set aside (or eliminate) his evidence $x_{3}$ relevant to the assertion that the store is open. But since $x_{5}$ was sent privately to Bob, ${ }^{1}$ Bob has no reason to believe that Charlie will doubt the evidence $x_{3}$ that the store is open. Therefore, while message $x_{5}$ causes Bob to eliminate his evidence $x_{3}$ relevant to the assertion that the store is open-and so to abandon his combined evidence $x_{1}$ and $x_{3}$ that Anne is bringing the cheese-Bob will nevertheless maintain his belief based on the combination of evidence $x_{2}$ and $x_{4}$ that Charlie still believes based on the combination of evidence $x_{1}$ and $x_{3}$ that Anne will bring the cheese.

\section{Introduction}

As indicated by our email example, this paper concerns reasoning about multi-agent communication and evidence elimination. Our work here is part of a larger project aimed at joining two areas of study: Justification Logic, ${ }^{2}$ a family of logics for reasoning about evidence and justification, and Dynamic Epistemic Logic, ${ }^{3}$ a family of logics for reasoning about multi-agent communication and belief. Before we discuss our rationale for seeking a combination of these theories, let us first say a few words about each of them in isolation.

Justification Logic has been promoted as a logic for reasoning about evidence and justification (Artemov 2008; Fitting 2009). The idea here is to remedy a deficiency found in the standard use of modal logic for reasoning about the justifications agents have for the beliefs that they possess. To illustrate this deficiency, consider a valid modal formula of the form $B_{i} \varphi \rightarrow B_{i} \psi$, where $B_{i}$ is a modal operator and each of $\varphi$ and $\psi$ are formulas. This formula, read, "if agent $i$ believes $\varphi$, then agent $i$ believes $\psi$," stipulates a certain connection between agent $i$ believing one thing, $\psi$, and agent

\footnotetext{
1 For simplicity, we assume that neither CC ("carbon copy") nor BCC ("blind carbon-copy") is possible. The recipients of a message are therefore those individuals mentioned in the "To" field.

2 See: Artemov (2001, 2008); Artemov and Nogina (2005); Fitting (2005, 2009); Kuznets (2008); Renne (2008a).

3 See: Baltag and Moss (2004); Baltag et al. (1998, 2008); Renne (2008b); van Benthem (2006) van Benthem et al. (2006); van Ditmarsch et al. (2007).
} 
$i$ believing another thing, $\varphi$. But we see that this formula does not provide a reason as to why agent $i$ 's belief in one thing follows whenever he or she has belief in another. The formula merely asserts that such conditional belief holds without providing any explanation as to why this is the case.

Justification Logic aims to better explicate this situation by first introducing structured syntactic objects called terms and then allowing us to form new formulas of the form $t:_{i} \varphi$ whenever $t$ is a term, $i$ is an agent, and $\varphi$ is a formula we have already formed. The idea is to identify the structure of the term $t$ with an abstract description of a derivation within a given theory of Justification Logic in a way that satisfies the Internalization Theorem (Artemov 2001): if $p$ is a proof of a theorem $\chi$ in the logic, then there is a systematic way to construct a term $u_{p}$ whose structure reflects the structure of $p$ such that $u_{p}:_{i} \chi$ is also a theorem of the logic. This provides us with a proof-based notion of evidence, in the sense that the appearance of a term $t$ in a theorem $t: i \varphi$ encodes a description as to why it is (according to the theory) that agent $i$ believes that $\varphi$ holds. This leads us to read the formula $t:_{i} \varphi$ as " $t$ is agent $i$ 's evidence that $\varphi$ is true."

Returning to the deficiency of modal logic, Justification Logic enables us to replace the statement $B_{i} \varphi \rightarrow B_{i} \psi$ of conditional (modal) belief with a more nuanced statement of conditional evidence-based belief: for a term $s_{t}$ built up from $t$, the formula $t:_{i} \varphi \rightarrow s_{t}:_{i} \psi$ says, "if $t$ is agent $i$ 's evidence for $\varphi$, then $s_{t}$ is agent $i$ 's evidence for $\psi$." Since $s_{t}$ is built up from $t$, we see that this tells us that in case agent $i$ has justification $t$ for his or her belief in $\varphi$, then agent $i$ may justify his or her belief in $\psi$ by inserting the argument $t$ into the appropriate places in the argument $s_{t}$, thereby yielding an argument supporting $\psi$. In this way, Justification Logics provide an in-language notion of proofbased evidence. To distinguish this notion of evidence from other notions of evidence, it may be helpful to think of proof-based evidence in the following intuitive way: to have proof-based evidence for $\varphi$ is to have deductive argumentation supporting $\varphi$.

One immediate limitation for the proof-based notion of evidence found in many Justification Logics is that it is static: in these theories, either agent $i$ has an argument supporting $\varphi$, in which case he or she has and will always have evidence for $\varphi$, or else agent $i$ does not have an argument supporting $\varphi$, in which case he or she does not and will never have evidence for $\varphi$. Such Justification Logics do not allow agents to learn new evidence, to forget old evidence, or to have a change of mind about the reliability of existing evidence. This is clearly at odds with everyday experience where, for example, agent $i$ may believe $\psi$ on the basis of an argument $s_{t}$ only to find out later that the evidence $t$ on which $s_{t}$ is crucially dependent is completely unreliable, thereby forcing agent $i$ to throw out both $t$ and $s_{t}$ and hence the belief in $\psi$ on the basis of $s_{t}$. What is missing from basic Justification Logics is a certain evidence dynamics, whereby certain basic pieces of evidence can change their status from "good" to "bad" (or the other way around).

A further criticism of the proof-based notion of evidence is that it neglects the intuitive social role that evidence plays as a vehicle for persuasion: if agent $i$ has an argument supporting $\varphi$ but agent $j$ does not, then agent $i$ ought to be able to tell agent $j$ of $i$ 's argument supporting $\varphi$, which might have the effect of convincing agent $j$ to believe $\varphi$. Here what is missing is a means of describing how evidence dynamics can be brought about as a result of communication. But note that the way in which this 
communication takes place must also be taken into account: $i$ 's argument might be told to $j$ in public, which then might also effect the beliefs of other agents who hear of $i$ 's argument; alternatively, $i$ 's argument may be told to $j$ in private, which would probably not effect the others' beliefs (though they might develop suspicions as to what $i$ and $j$ are talking about if they see $i$ and $j$ depart for a closed-door meeting).

One framework that elegantly describes a wide range of multi-agent communications-whether public or private, with or without deception, having or not having suspicion, and so on-is the framework of Dynamic Epistemic Logic. In Dynamic Epistemic Logic, a communication is modeled using what we call an update frame, which is a finite Kripke frame ${ }^{4}$ whose worlds have been labeled by formulas. The idea, developed by Baltag, Moss, and Solecki (BMS) (Baltag and Moss 2004; Baltag et al. 1998) based on work on public announcements by Plaza $(1989,2007)$ and by Gerbrandy and Groeneveld (Gerbrandy 1999; Gerbrandy and Groeneveld 1997), is that each world $w$ in an update frame represents a possible communication of the formula $\chi_{w}$ that labels $w$. The structure of the arrows in the update frame describes the agents' conditional uncertainties as to which communication is actually taking place: in case there is an $i$-arrow from world $w$ to world $w^{\prime}$, then agent $i$ will think it possible that the formula $\chi_{w^{\prime}}$ is communicated if it is the case that the formula $\chi_{w}$ is actually communicated. This allows us to use update frames to represent a wide variety of communications.

As an example, for a nonempty finite set $\mathscr{A}$ of agents, the update frame

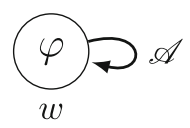

represents the public announcement of $\varphi$. If $\chi_{w}=\varphi$ is actually communicated, then each agent $i \in \mathscr{A}$ thinks that the only formula that can possibly be communicated is the formula $\chi_{w}=\varphi$ itself. ${ }^{5}$ Thus to have such a communication occur is to make it the case that every agent will simultaneously come to know $\varphi$, which is intuitively what happens when a public announcement of $\varphi$ occurs. Of course, the update frame above for the public announcement of $\varphi$ also says something about higher-order knowledge: since there is a unique $i$-arrow from $w$ to $w$ for an agent $i \in \mathscr{A}$ and since there is also a unique $j$-arrow from $w$ to $w$ for another agent $j \in \mathscr{A}, j$ knows that $i$ knows that $\varphi$ is to be communicated. After all, at the unique world $w$ that $j$ considers possible, agent $i$ knows that $\chi_{w}$ is the formula that is communicated. It is not too hard to see that the update frame above describes a situation in which it is common knowledge that $\varphi$ is communicated; that is, each agent knows that $\varphi$ is communicated, each agent knows that each agent knows that $\varphi$ is communicated, each agent knows that each agent knows that each agent knows that $\varphi$ is communicated, and so on for any finite

\footnotetext{
4 A finite Kripke frame is a finite directed graph whose nodes are called "worlds" and whose edges are labeled by agents' names. We will call the edges of a finite Kripke frame "arrows" and we will use the phrase " $X$-arrow" to refer to an arrow that is labeled by $X$.

5 We adopt the following drawing convention for update frames: for a (possibly empty) set $S$ of agents, if a drawing of an update frame contains an $S$-arrow from a world $w$ to a world $w^{\prime}$, then what is meant is that for each agent $a \in S$, there is an $a$-arrow from $w$ to $w^{\prime}$.
} 
number of occurrences of the phrase "each agent knows that" appearing before the phrase " $\varphi$ is communicated."

As another example, the update frame

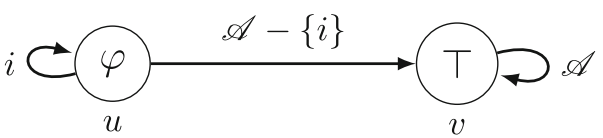

represents the private announcement of $\varphi$ to agent $i$, since in case $\chi_{u}=\varphi$ is the formula that is actually communicated, the unique $i$-arrow from $u$ to $u$ says that $i$ believes that the only formula that can possibly be communicated is the formula $\chi_{u}=\varphi$, whereas the unique $j$-arrow from $u$ to $v$ for each $j \in \mathscr{A}-\{i\}$ says that any other agent $j$ believes that the formula $\chi_{v}=\top$ (the propositional constant for truth, which conveys no new information) was communicated. ${ }^{6}$ Thus if $\varphi$ is actually communicated, then the information about $\varphi$ is sent to agent $i$ while all the other agents gain no new information. This is intuitively what it is to have a private announcement of $\varphi$ to agent $i$.

Using symbols to denote update frames, Dynamic Epistemic Logic allows us to form new formulas of the form $[U, u] \varphi$, where $U$ is a symbol for an update frame, $u$ is a symbol naming a world in the update frame $U$ (here $u$ indicates that the formula $\chi_{u}$ is what is actually communicated), and $\varphi$ is a formula that has already been formed. The formula $[U, u] \varphi$ then says, "after the update $(U, u)$, where the formula $\chi_{u}$ is actually communicated, we have that $\varphi$ is true." (Note that we use the words "update" and "communication" interchangeably because very complicated update frames are better thought of as generalized "informational updates" due to the fact that there is not always an intuitive, everyday communicative type corresponding to each complex update frame; see the discussion in Renne (2006).) Since the formula $\varphi$ occurring in $[U, u] \varphi$ can be a formula describing various agents' beliefs, we see that Dynamic Epistemic Logic can be used to describe how communications affect the beliefs of agents; for example, $[U, u] B_{i} \psi$ says that agent $i$ believes $\psi$ after the occurrence of the update $(U, u)$.

While a communication can affect agent belief, the language of Dynamic Epistemic Logic - itself an extension of the language of modal logic_-does not provide us with the means to describe reasons for such changes in agent belief. To illustrate, let $p$ be a propositional letter and then consider a valid formula of the form $p \rightarrow[U, u] B_{i} p$ ("if $p$ is true, then, after update $(U, u)$, agent $i$ believes $p$ "). This formula stipulates a connection between the occurrence of the update $(U, u)$ and agent $i$ coming to believe the atom $p$. In essence, the content of the update $(U, u)$ brings about this change in agent $i$ 's belief, though the reason why this particular change in belief is brought about is left unstated - the formula says only that this communication brings about $i$ 's change in belief for some reason. (This is similar to the case of modal logic itself,

\footnotetext{
6 More precisely: the $\mathscr{A}$-arrow from $v$ to $v$ makes it so that in case $\chi_{u}=\varphi$ is the formula that is actually communicated, each of the non- $i$ agents mistakenly believes that it is common belief that $T$ is communicated (and so no new information is conveyed). So while $i$ learns the new information $\varphi$ - this due to the $i$-arrow from $u$ to $u$ - the other agents have the mistaken common belief that no new information has been communicated.
} 
where the formula $B_{i} \varphi \rightarrow B_{i} \psi$ does not say why it is that $i$ 's belief in $\psi$ follows whenever he or she believes $\varphi$.) Indeed, despite the fact that an explanation based on the structure of $U$ justifies this change in $i$ 's belief, this explanation is not expressible within the language itself. So for example, if $U$ is the public announcement of $p$, then the explanation of why $(U, u)$ causes $i$ to believe $p$ would go something like "since a public announcement of a fact causes all agents to come to learn that fact, agent $i$ will come to believe the fact $p$ after it is publicly announced"; however, such an explanation cannot be formulated within the language. Furthermore, if we suppose that another agent $j$ believes that update $(U, u)$ will cause agent $i$ to come to believe the fact $p$,

$$
B_{j}\left(p \rightarrow[U, u] B_{i} p\right),
$$

then, just as in modal logic, there is no way for the language to describe agent $j$ 's justification for this belief. In short, the language is not only lacking the capacity to express the agents' reasons for holding their beliefs (as is the case with modal logic) but it is also unable to express the agents' reasons for changing their beliefs.

To summarize what we have said thus far, we have argued that each of Justification Logic and Dynamic Epistemic Logic would be better suited for modeling, describing, and studying rational agency if it had additional features. In the case of Justification Logic, we have argued for the ability to handle evidence dynamics, taking into account the social role that evidence plays as a vehicle for persuasion in various kinds of communication, be it public or private, with or without deception, having or not having suspicion, and so on. In the case of Dynamic Epistemic Logic, we have argued for an in-language account of evidence so that agents can provide justifications not only as to why they hold certain beliefs but also as to why they would change these beliefs. We thus think it natural to look for a combined framework-something that might eventually be called Dynamic Justification Logic - that brings together the complementary strengths from each of Dynamic Epistemic Logic and Justification Logic so as to address each area's respective weakness.

In this paper, we begin the project of Dynamic Justification Logic by proposing a theory called JLCE that combines multi-agent communication and belief from Dynamic Epistemic Logic with a new multi-agent Justification Logic for reasoning about evidence and justification. JLCE, the theory of Justification Logic with Communication and Elimination, allows us to reason not only about multi-agent belief dynamics arising as the result of Dynamic Epistemic Logic-style communicationshereafter called (multi-agent) communications - but also about a kind of multi-agent evidence dynamics we call evidence elimination, whereby a piece of evidence $t$ relevant to a particular claim is to be set aside as part of a multi-agent communication whose content effectively undermines the basic pieces of evidence that make up $t$. Our email example indicates one of the many kinds of multi-agent evidence eliminations that JLCE can reason about: with Bob's basic piece of evidence $x_{3}$ undermined, his more complex evidence obtained by combining the rotten piece of evidence $x_{3}$ with another piece of evidence $x_{1}$ is also undermined.

After defining the language, axiomatics, and semantics of JLCE, we will show how this theory can be used to formalize the evidence elimination from our email example. Along the way, we will prove a number of results about JLCE. 


\section{Syntax}

Our language, called UL (for "update language"), is used to reason about multi-agent communication and evidence elimination for a finite nonempty set $\mathscr{A}$ of rational agents. The atoms of UL are given as follows.

Definition 3.1 We define the sets

$$
\begin{aligned}
& \mathscr{P}:=\left\{p_{k} \mid k \in \mathbb{N}\right\} \text { of propositional letters, } \\
& \mathscr{C}:=\left\{c_{k} \mid k \in \mathbb{N}\right\} \text { of constants, and } \\
& \mathscr{V}:=\left\{x_{k} \mid k \in \mathbb{N}\right\} \text { of variables. }
\end{aligned}
$$

To define the full language of UL, we introduce the notion of update frame. Update frames are an extension of the "action models" found in the Dynamic Epistemic Logic literature (Baltag et al. 2008; Renne 2008b; van Ditmarsch et al. 2007).

Definition 3.2 Given a nonempty set $F$ of formulas, to say that $U$ is an $F$-update frame means that $U$ is a tuple $\left(W^{U}, R^{U}, \mathrm{p}^{U}, \mathrm{v}^{U}\right)$ whose components satisfy the following.

- $W^{U}$ is a nonempty finite set of worlds (in $U$ ).

- $R^{U}: \mathscr{A} \rightarrow \wp\left(W^{U} \times W^{U}\right)$ assigns a transitive binary relation $R_{i}^{U}$ to each agent $i \in \mathscr{A}^{7}$

- $\mathrm{p}^{U}: W^{U} \rightarrow F$ labels worlds with formulas.

- $\mathrm{v}^{U}: W^{U} \rightarrow \wp(\mathscr{V} \times \mathscr{A} \times F)$ labels worlds with finite sets of variable-agentformula triples $(x, i, \varphi) .^{8}$

An $F$-update frame $U$ and a world $u \in W^{U}$ form a pointed $F$-update frame with point $u$. For a set $F^{\prime}$ of formulas, we write $\mathscr{U}\left(F^{\prime}\right)$ to denote the set of pointed $F^{\prime}$ update frames.

Pointed update frames are used to describe communications along with eliminations of relevant evidence. Informally speaking, to say that tis relevant for agent $i$ to $\varphi$ means that $i$ considers evidence $t$ to be probative for $\varphi$ - that is, $i$ believes that $t$ generally tends to demonstrate or prove $\varphi$ - though $i$ need not be persuaded to believe $\varphi$ on the basis of evidence $t$. So to have $i$ take evidence $t$ as relevant to $\varphi$ is just to say that $i$ considers $t$ to be admissible as evidence for $\varphi$, regardless of whether this admissibility also comes with belief. We will write " $t \gg_{i} \varphi$ " to mean that $t$ is relevant for $i$ to $\varphi$. Note that agent $i$ may consider many different pieces of evidence to be relevant to a single assertion $\varphi$. The statement $t \gg_{i} \varphi$ merely asserts that $t$ is among the possibly many pieces of evidence that are relevant to $\varphi$.

When agent $i$ has a piece of evidence $t$ relevant to $\varphi$ and agent $i$ also believes that $\varphi$ is true, then we will say that $t$ is agent $i$ 's evidence for believing $\varphi$ and we will write

\footnotetext{
7 Our reasons for taking $R_{i}^{U}$ transitive are for convenience only. We shall discuss this point later in Remark 3.13.

${ }^{8}$ Finiteness ensures that update frames can in principle be written out (using an appropriate syntactic encoding) and that a forthcoming theory based on update frames (the $U$-calculus, Definition 3.5 ) is decidable.
} 
" $t:_{i} \varphi$ ". So we have two notions of evidence: the weaker notion $t \gg_{i} \varphi$ of relevant evidence and the stronger notion $t:_{i} \varphi$ of relevant evidence plus belief.

Pointed update frames $(U, u)$ will be used to describe an update, which is a multiagent communication. A pointed update frame $(U, u)$ describes the communication of the formula $\mathrm{p}^{U}(u)$ within a collection $\left\{\mathrm{p}^{U}(v) \mid v \in W^{U}\right\}$ of formulas that might be communicated. So while the update $(U, u)$ actually communicates the formula $\mathrm{p}^{U}(u)$, the agents have uncertainty as to which communication actually occurs according to the structure of the Kripke frame $\left(W^{U}, R^{U}\right)$ underlying $U$ and the way in which $\mathrm{p}^{U}$ labels worlds in $U$ by formulas. We explained this in greater detail above in the introduction, where we gave examples of two update frames, one for the public announcement of $\varphi$ and one for the private announcement of $\varphi$ to agent $i$.

Our contribution in this paper is to add a notion of multi-agent evidence elimination to the above-described BMS-picture. We thus view each world in an update frame $U$ as constituting a possible communication and elimination: for an update $(U, u)$, a variable $x \in \mathscr{V}$, an agent $i \in \mathscr{A}$, and an assertion $\varphi$, if $(x, i, \varphi) \in \mathrm{v}^{U}(u)$, then the communication and elimination possibility $u$ simultaneously communicates $\mathrm{p}^{U}(u)$ and eliminates agent $i$ 's evidence $x$ relevant to $\varphi$, which has the effect of falsifying $x \gg_{i} \varphi$. But an elimination of a variable $x$ relevant to an assertion can have consequences for other pieces of evidence $s_{x}$ that are built up from $x$. We saw this in our email example: Anne's final email $x_{5}$ eliminated Bob's basic piece of evidence $x_{3}$ relevant to the store being open, and this elimination itself triggered an elimination of Bob's combined evidence $x_{1}$ and $x_{3}$ that Anne is bringing the cheese.

Definition 3.3 $\mathscr{G}_{0}$ is the following grammar.

$$
\begin{aligned}
t::= & c|x| t \cdot \varphi t|t+t| ! t \\
\varphi::= & \perp|p| \varphi \rightarrow \varphi\left|B_{i} \varphi\right| t \gg_{i} \varphi \\
& c \in \mathscr{C}, x \in \mathscr{V}, p \in \mathscr{P}, i \in \mathscr{A}
\end{aligned}
$$

Let $\mathscr{T}_{0}$ be the set of expressions built using $t$ as a start symbol in grammar $\mathscr{G}_{0}$, and let $\mathscr{F}_{0}$ be the set of expressions built using $\varphi$ as a start symbol in grammar $\mathscr{G}_{0}$. Then, whenever the pair $\left(\mathscr{T}_{k}, \mathscr{F}_{k}\right)$ is defined, we define grammar $\mathscr{G}_{k+1}$ as follows.

$$
\begin{aligned}
t::= & s\left|t \cdot{ }_{\varphi} t\right| t+t \mid ! t \\
\varphi::= & \psi|\varphi \rightarrow \varphi| B_{i} \varphi\left|t \gg_{i} \varphi\right|[U, u] \varphi \\
& s \in \mathscr{T}_{k}, \psi \in \mathscr{F}_{k}, i \in \mathscr{A},(U, u) \in \mathscr{U}\left(\mathscr{F}_{k}\right)
\end{aligned}
$$

Let $\mathscr{T}_{k+1}$ be the set of expressions built using $t$ as a start symbol in grammar $\mathscr{G}_{k+1}$, and let $\mathscr{F}_{k+1}$ be the set of expressions built using $\varphi$ as a start symbol in grammar $\mathscr{G}_{k+1}$. Finally, we define the set $\mathscr{T}:=\bigcup_{k \in \mathbb{N}} \mathscr{T}_{k}$ of UL-terms (also called terms) and the set $\mathscr{F}:=\bigcup_{k \in \mathbb{N}} \mathscr{F}_{k}$ of UL-formulas (also called formulas). We adopt the usual abbreviations for other logical connectives. The update language, written UL, is the pair $(\mathscr{T}, \mathscr{F})$ consisting of the set $\mathscr{T}$ of terms and the set $\mathscr{F}$ of formulas. We define the set $\mathscr{U}:=\mathscr{U}(\mathscr{F})$ of pointed UL-update frames (also called pointed update frames).

Definition $3.4 t:_{i} \varphi$ abbreviates $\left(t \gg_{i} \varphi\right) \wedge B_{i} \varphi$. 
The constants and variables together constitute the atomic terms. The symbol $B_{i}$ is called an $i$-belief modal (or simply a belief modal), the symbol $\gg_{i}$ is called an $i$-arrow (or simply an arrow), the symbol $:_{i}$ is called an $i$-colon (or simply a colon), and the symbol $[U, u]$ is called an update modal. There are a number of interesting sub-languages of UL; in particular, the basic fragment is the pair $\left(\mathscr{T}_{b}, \mathscr{F}_{b}\right)$ obtained from $\left(\mathscr{T}_{0}, \mathscr{F}_{0}\right)$ by restricting formulas containing belief modals or arrows to the form $t: i \varphi$. We assign the following informal readings to the key formulas in UL.

- $B_{i} \varphi$ is read, "agent $i$ believes $\varphi$."

- $t \gg_{i} \varphi$ is read, " $t$ is agent $i$ 's evidence relevant to $\varphi$."

- $t:_{i} \varphi$ is read, " $t$ is agent $i$ 's evidence for believing $\varphi . "$

- $[U, u] \varphi$ is read, "after update $(U, u)$, formula $\varphi$ is true."

Each term describes a way of obtaining derived evidence from given evidence through the use of logical principles; let us discuss this now. The constants $c \in \mathscr{C}$ play a special role as evidence relevant to the axioms of our theory, and the variables $x \in \mathscr{V}$ are used as contingent relevant evidence that may be directly affected by an evidence elimination. The operation $\cdot{ }_{\varphi}$ takes evidence $t$ relevant to an implication $\varphi \rightarrow \psi$ and evidence $s$ relevant to the antecedent $\varphi$ and produces the evidence $t \cdot{ }_{\varphi} s$ relevant to the consequent $\psi$, in accord with the rule of Modus Ponens. The operation + is the union of pieces of relevant evidence: $t+s$ is relevant evidence for anything for which one or both of $t$ and $s$ is relevant evidence. The operation! ("bang") is an evidence checker: in case $t$ is relevant evidence for $\varphi$, then ! $t$ ("bang $t$ ") checks that $t$ is evidence for $\varphi$.

Definition 3.5 Given an update frame $U$, the $U$-calculus is defined in Fig. 1 .

The $U$-calculus will be used to describe the effect evidence eliminations have on evidence relevance in the following way: the update $(U, u)$ eliminates agent $i$ 's evidence $t$ relevant to $\varphi$-meaning that $t \gg_{i} \varphi$ will be false after update $(U, u)$-if and only if $U, u \vdash(t, i, \varphi)$ is derivable in the $U$-calculus.

Remark 3.6 (Variable-Driven Elimination) We observe that our formulation of the $U$-calculus in Fig. 1 restricts evidence elimination in such a way that whenever update $(U, u)$ eliminates agent $i$ 's evidence $t$ relevant to $\varphi$-thereby falsifying $t \gg_{i} \varphi$-this

$$
\begin{gathered}
\text { RULES } \\
\frac{\left(x_{k}, i, \varphi\right) \in \mathrm{v}^{U}(u)}{U, u \vdash\left(x_{k}, i, \varphi\right)}(\mathrm{EV}) \\
\frac{U, u \vdash(t, i, \varphi \rightarrow \psi)}{U, u \vdash(t \cdot \varphi s, i, \psi)}(\mathrm{EAL}) \quad \frac{U, u \vdash(s, i, \varphi)}{U, u \vdash(t \cdot \varphi, i, \psi)}(\mathrm{EAR}) \\
\frac{U \vdash(t, i, \varphi) \quad U, u \vdash(s, i, \varphi)}{U, u \vdash(t+s, i, \varphi)}(\mathrm{ES}) \frac{U, u \vdash(t, i, \varphi)}{U, u \vdash\left(! t, i, t:_{i} \varphi\right)}(\mathrm{EC}) \\
\frac{U, u^{\prime} \vdash\left(x_{k}, i, \varphi\right)}{U, u \vdash\left(x_{k}, i, \varphi\right)} u R_{i}^{U} u^{\prime} \\
\text { (EM) }
\end{gathered}
$$

Fig. 1 The $U$-calculus 
elimination can be traced back to an elimination of one or more variables $x \in \mathscr{V}$ as relevant evidence for agent $i$. This restriction, which we call variable-driven elimination, guarantees that whenever a formula of the form $s \gg_{i} \psi$ is derivable in our forthcoming theory JLCE (Definition 3.12), it will not be possible for an update $\left(U^{\prime}, u^{\prime}\right)$ to eliminate $s$ as agent $i$ 's evidence relevant to $\psi$. Variable-driven elimination therefore maintains a property of elimination consistency: to say that $s \gg_{i} \psi$ is derivable in our forthcoming theory JLCE means that $s$ is always agent $i$ 's evidence relevant to $\psi$, and hence no elimination can eliminate $s$ as agent $i$ 's evidence relevant to $\psi$. While the author conjectures that it is possible to develop an alternative to the $U$-calculus that provides for elimination consistency under a condition weaker than that of variabledriven elimination, allowing eliminations to be traced back to a term $t \in \mathscr{T}$ that need not be a variable, various syntactic consistency checks would need to be imposed on the structure of update-frame-eliminable terms in order to maintain elimination consistency. In the interest of simplicity in a first paper on evidence elimination in Justification Logic, the author has gone the route of variable-driven elimination. This may seem problematic: in everyday life, one often discovers a problem with his or her reasoning only after it has gone on for some time and then suddenly faces a contradiction. This suggests that one should eliminate the more complex piece of evidence $s$ directly and then see what consequences this has not only for the various pieces of complex evidence that make use of $s$ but also those pieces of simpler evidence that themselves make up $s$. Put another way, what is suggested is a general theory of evidence-based Belief Revision, in which an agent reasons in a step-by-step manner according to his or her evidence, adjusting this evidence on-the-fly whenever contradictions are encountered or new information comes in. While this is an extremely interesting and promising line of work, the scope of this study is too large for the purposes of a single paper.

The goal of this paper is to provide a basic account of the way in which complex pieces of evidence are affected by changes in basic pieces of evidence. So evidence elimination is not at all a general account of evidence-based Belief Revision but instead an account of part of an adversarial argumentative process that plays an important role in the overall evidence-based Belief Revision picture. In particular, this process is one in which an agent's reasoning is successfully undermined by an attack on the premises underlying that reasoning. To illustrate, think of the friends in our email example as employees of a catering company managed by Bob and assume that an angry client Daisy sues Bob in court, alleging that the employees of the company ruined her party by providing crackers without any cheese, and this was due to the manager Bob's negligence. Bob might show emails $x_{1}$ through $x_{4}$ to the court to argue that he was not negligent. But when Daisy manages to obtain a copy of email $x_{5}$ and shows this to the court, thereby eliminating Bob's evidence that he did not know of any problem his employees might have in delivering both the crackers and the cheese, the court will presumably side with Daisy. So we see how evidence elimination is about this fundamental adversarial process of undermining an agent's reasoning by attacking the premises. But while this process is very important to a general theory of evidencebased Belief Revision, it is merely one way in which evidence changes in light of new information within a generalized framework of evidence-based Belief Revision. Other changes that directly affect more complex pieces of evidence need to be studied 
as well, and future work in Dynamic Justification Logic will aim toward establishing a general account of evidence-based Belief Revision that does just this. In the meantime, the work in the present paper shall stick to the study of variable-based evidence elimination.

Given $(U, u)$ and $(t, i, \varphi)$, either $U, u \vdash(t, i, \varphi)$ or $U, u \nvdash(t, i, \varphi)$, and determining which of these is the case is a decidable question. Further, the $U$-calculus satisfies the following anti-monotonicity property.

Lemma 3.7 (Anti-Monotonicity) $U, u^{\prime} \vdash(t, i, \psi)$ and $u R_{i}^{U} u^{\prime}$ together imply that $U, u \vdash(t, i, \psi)$.

Proof By a straightforward induction on the length of the derivation of $U, u^{\prime} \vdash$ $(t, i, \psi)$.

To state the axiomatics, we define a notion of composition for update frames that allows us to take pointed update frames $(U, u)$ and $\left(U^{\prime}, u^{\prime}\right)$ and build a pointed update frame $\left(U \circ U^{\prime},\left(u, u^{\prime}\right)\right)$ whose execution has the same effect as the sequential execution of update $(U, u)$ followed by update $\left(U^{\prime}, u^{\prime}\right)$.

Notation 3.8 (Functions on Pairs) We shall often write " $f(a, b)$ " instead of " $f((a, b))$ " when we wish to denote the value of function $f$ on the pair $(a, b)$; our intended meaning will be clear from context.

Definition 3.9 To define the composition $U \circ U^{\prime}$, we use standard definitions from Dynamic Epistemic Logic Baltag et al. (2008); Renne (2008b); van Ditmarsch et al. (2007) for the first three components: set $W^{U \circ U^{\prime}}:=W^{U} \times W^{U^{\prime}}$, allow $\left(u, u^{\prime}\right) R_{i}^{U \circ U^{\prime}}\left(v, v^{\prime}\right)$ if and only if $u R_{i}^{U} v$ and $u^{\prime} R_{i}^{U^{\prime}} v^{\prime}$, and set $\mathrm{p}^{U \circ U^{\prime}}\left(u, u^{\prime}\right):=\neg[U, u]$ $\neg \mathrm{p}^{U^{\prime}}\left(u^{\prime}\right)$. To define the final component $\mathrm{v}^{U \circ U^{\prime}}$, we set $\mathrm{v}^{U \circ U^{\prime}}\left(u, u^{\prime}\right):=\mathrm{v}^{U}(u) \cup$ $\mathrm{v}^{U^{\prime}}\left(u^{\prime}\right)$.

Theorem 3.10 (Composition Correctness) If $U$ and $U^{\prime}$ are update frames, then so is $U \circ U^{\prime}$.

Proof It suffices to verify that $R_{i}^{U \circ U^{\prime}}$ is transitive for each $i \in \mathscr{A}$, but this follows by the definition of $R_{i}^{U \circ U^{\prime}}$ and the fact that each of $R_{i}^{U}$ and $R_{i}^{U^{\prime}}$ is transitive.

The definition of composition ensures that $\left(U \circ U^{\prime},\left(u, u^{\prime}\right)\right)$ eliminates agent $i$ 's evidence $t$ relevant to the assertion that $\varphi$ if and only if at least one of $(U, u)$ or $\left(U^{\prime}, u^{\prime}\right)$ eliminates $i$ 's evidence $t$ relevant to the assertion that $\varphi$.

Lemma 3.11 (Composition) Given update frames $U$ and $U^{\prime}$, the following statements are equivalent.

- $U \circ U^{\prime},\left(u, u^{\prime}\right) \vdash(t, i, \varphi)$.

- $U, u \vdash(t, i, \varphi)$ or $U^{\prime}, u^{\prime} \vdash(t, i, \varphi)$.

Proof By induction on the construction of $t$. The base case $t \in \mathscr{C}$ follows immediately and the base case $t \in \mathscr{V}$ follows because $\mathrm{v}^{U \circ U^{\prime}}\left(u, u^{\prime}\right)=\mathrm{v}^{U}(u) \cup \mathrm{v}^{U^{\prime}}\left(u^{\prime}\right)$. The induction cases $t=s_{1} \cdot{ }_{\varphi} s_{2}, t=s_{1}+s_{2}$, and $t=! s$ follow by the induction hypothesis and respective use of rules EAL and EAR, ES, and EC (Fig. 1). 
Finally, we state in two parts the axioms and rules of our theory of Justification Logic with Communication and Elimination, written JLCE. The first part is the axiomatic theory AX (Fig. 2), and the second part is our theory JLCE itself (Fig. 3).

Definition 3.12 The theory $A X$ is defined in Fig. 2. Regarding Axiom U3, we stipulate that a conjunction $\bigwedge_{u R_{i}^{U} v} \chi_{v}$ ranging over the set $S:=\left\{v \in W^{U} \mid u R_{i}^{U} v\right\}$ is to be identified with a fixed tautology denoted by "T" whenever $S=\emptyset$. The theory JLCE is defined in Fig. 3. The theory JLCE* consists of Rules AX and MP from Fig. 3.

We will see later that the theories JLCE and JLCE* derive the same theorems. This result will be useful: while our primary interest is in the full theory JLCE, it is sometimes easier to prove a given result using the simpler theory JLCE*.

\section{BELIEF/EVIDENCE SCHEMES}

CL. Classical propositional tautologies

B1. $\quad B_{i}(\varphi \rightarrow \psi) \rightarrow\left(B_{i} \varphi \rightarrow B_{i} \psi\right)$

B2. $B_{i} \varphi \rightarrow B_{i} B_{i} \varphi$

E1. $t \gg_{i}(\varphi \rightarrow \psi) \rightarrow\left(\left(s \gg_{i} \varphi\right) \rightarrow(t \cdot \varphi s) \gg_{i} \psi\right)$

E2. $\quad\left(t \gg_{i} \varphi\right) \rightarrow(t+s) \gg_{i} \varphi$

$\left(s \gg_{i} \varphi\right) \rightarrow(t+s) \gg_{i} \varphi$

E3. $\left(t \gg_{i} \varphi\right) \rightarrow ! t \gg_{i}\left(t:_{i} \varphi\right)$

E4. $\quad\left(t \gg_{i} \varphi\right) \rightarrow B_{i}\left(t \gg_{i} \varphi\right)$

\section{ELIMINATION/UPDATE SCHEMES}

U1. $\quad[U, u] q \leftrightarrow\left(\mathrm{p}^{U}(u) \rightarrow q\right)$ if $q \in \mathscr{P} \cup\{\perp\}$

U2. $[U, u](\varphi \rightarrow \psi) \leftrightarrow([U, u] \varphi \rightarrow[U, u] \psi)$

U3. $[U, u] B_{i} \varphi \leftrightarrow\left(\mathrm{p}^{U}(u) \rightarrow \bigwedge_{u R_{i}^{U} v} B_{i}[U, v] \varphi\right)$

U4. $\quad[U, u]\left(t \gg_{i} \varphi\right) \leftrightarrow \neg \mathrm{p}^{U}(u)$ if $U, u \vdash(t, i, \varphi)$

$[U, u]\left(t \gg_{i} \varphi\right) \leftrightarrow\left(\mathrm{p}^{U}(u) \rightarrow t \gg_{i} \varphi\right)$ if $U, u \nvdash(t, i, \varphi)$

U5. $\quad[U, u]\left[U^{\prime}, u^{\prime}\right] \varphi \leftrightarrow\left[U \circ U^{\prime},\left(u, u^{\prime}\right)\right] \varphi$

$$
\text { RULE: } \frac{\varphi}{c_{k}: i \varphi}(\mathrm{CN})
$$

Fig. 2 The theory $A X$

\section{RULES}

$$
\begin{array}{cl}
\frac{\mathrm{AX} \vdash \varphi}{\varphi}(\mathrm{AX}) & \frac{\varphi \rightarrow \psi \varphi}{\psi}(\mathrm{MP}) \\
\frac{\varphi}{B_{i} \varphi}(\mathrm{BN}) & \frac{\varphi}{[U, u] \varphi}(\mathrm{UN})
\end{array}
$$

Fig. 3 The theory JLCE 
Remark 3.13 We observe two points. First, in the statement of Axiom U4 (Fig. 2), we have offloaded to the $U$-calculus the work of determining the particular form the axiom should take for a given triple $(t, i, \varphi)$. It is possible to eliminate this reliance on an external theory by including new formulas in the language that allow us to embed the $U$-calculus into the overall theory. See Renne (2011) for details on how this is done in a theory of simple evidence elimination for Justification Logic. The reason we have offloaded the work in this way in this paper is to save space and to simplify the presentation of the axiomatics.

Second, we have assumed that agent belief is governed by the modal theory K4 and hence that agent belief is introspective (meaning it satisfies Axiom B2, which says that if agent $i$ believes something, then she believes she believes it). While other choices are possible in Justification Logic Kuznets (2008); Renne (2008a), they would introduce complications here that would distract us from the focus of this paper: multi-agent communication and evidence elimination.

Notation 3.14 (Provable Equivalence $\varphi \Leftrightarrow \varphi^{\prime}$ and $\varphi \Leftrightarrow{ }^{*} \varphi^{\prime}$ ) We write $\varphi \Leftrightarrow \varphi^{\prime}$ to mean that $\vdash \varphi \Leftrightarrow \varphi^{\prime}$, and we write $\varphi \Leftrightarrow{ }^{*} \varphi^{\prime}$ to mean that $\vdash^{*} \varphi \Leftrightarrow \varphi^{\prime}$. Given $n \in \mathbb{N}$, we write

$$
\varphi_{0} \Leftrightarrow \varphi_{1} \Leftrightarrow \varphi_{2} \Leftrightarrow \cdots \Leftrightarrow \varphi_{n}
$$

to mean that we have $\varphi_{i} \Leftrightarrow \varphi_{i+1}$ for each $i \in \mathbb{N}$ with $i<n$. Similarly, given $n \in \mathbb{N}$, we write (1) with each " $\Leftrightarrow$ " replaced by " $\Leftrightarrow$ *" to mean that we have $\varphi_{i} \Leftrightarrow{ }^{*} \varphi_{i+1}$ for each $i \in \mathbb{N}$ with $i<n$.

\section{The internalization theorem}

Artemov (2001) first identified a key result of single-agent Justification Logics (where $|\mathscr{A}|=1$ ) called the property of internalization: if $\mathscr{A}=\{a\}$, then for each theorem $\varphi$, there is a term $t$ such that $t:_{a} \varphi$ is also a theorem. Internalization specifies the way in which a Justification Logic allows us to describe proofs of the theory using terms, which bolsters our reading of terms as pieces of evidence for the formulas that they label. In this section, we will prove the internalization property for JLCE. To do this, we will show first that JLCE* has internalization (this is the forthcoming Lemma 4.2) and then prove that JLCE* and JLCE derive the same theorems (this is the forthcoming Theorem 4.13). The proofs of these results require quite a bit of work, so we shall proceed incrementally. We begin with a lemma that shows that in JLCE* , evidence — and not just relevant evidence—is closed under the term-forming operations $\cdot \varphi$ and + .

Lemma 4.1 We have each of the following.

1. $\vdash^{*} t:_{i}(\varphi \rightarrow \psi) \rightarrow\left(\left(s:_{i} \varphi\right) \rightarrow\left(t \cdot{ }_{\varphi} s\right):_{i} \psi\right)$.

2. $\vdash^{*}\left(\left(t:_{i} \varphi\right) \vee\left(s:_{i} \varphi\right)\right) \rightarrow(t+s):_{i} \varphi$.

Proof Using PR to denote use of classical propositional reasoning, the proofs appear in Figs. 4 and 5. 


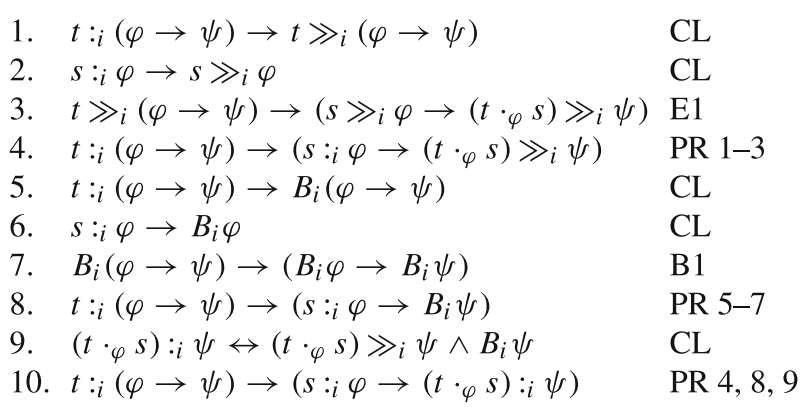

Fig. 4 Proof that $\vdash^{*} t:_{i}(\varphi \rightarrow \psi) \rightarrow\left(\left(s:_{i} \varphi\right) \rightarrow(t \cdot \varphi s):_{i} \psi\right)$
1. $t:_{i} \varphi \rightarrow t \gg_{i} \varphi$
CL
2. $t \gg_{i} \varphi \rightarrow(t+s) \gg_{i} \varphi$
E2
3. $t:_{i} \varphi \rightarrow B_{i} \varphi$
$\mathrm{CL}$
4. $(t+s):_{i} \varphi \leftrightarrow(t+s) \gg_{i} \varphi \wedge B_{i} \varphi \mathrm{CL}$
5. $t:_{i} \varphi \rightarrow(t+s):_{i} \varphi$
PR 1-4
6. $s:_{i} \varphi \rightarrow s \gg_{i} \varphi$
CL
7. $s \gg_{i} \varphi \rightarrow(t+s) \gg_{i} \varphi$
E2
8. $s:_{i} \varphi \rightarrow B_{i} \varphi$
CL
9. $s:_{i} \varphi \rightarrow(t+s):_{i} \varphi$
PR 4, 6-8
10. $\left(\left(t:_{i} \varphi\right) \vee\left(s:_{i} \varphi\right)\right) \rightarrow(t+s):_{i} \varphi$
PR 5, 9

Fig. 5 Proof that $\vdash^{*}\left(\left(t:_{i} \varphi\right) \vee\left(s:_{i} \varphi\right)\right) \rightarrow(t+s):_{i} \varphi$

Lemma 4.2 (JLCE* Internalization; (Artemov 2001; Renne 2008a)) If $\vdash^{*} \varphi$ and $i \in \mathscr{A}$, then there there is a term $t \in \mathscr{T}$ such that $\vdash^{*} t:_{i} \varphi$.

Proof This argument is a straightforward adaptation of the standard proof of Artemov's original internalization result Artemov (2001). Choosing $i \in \mathscr{A}$, the proof is by induction on the length of JLCE* derivations. If the last step of the proof was Rule $\mathrm{AX}$, then set $t:=c_{0}$ and observe that $\vdash^{*} c_{0}:_{i} \varphi$ by Rule AX. If the last step of the proof was Rule MP from premises $\psi \rightarrow \varphi$ and $\psi$, then it follows by the induction hypothesis that there is a term $s_{1} \in \mathscr{T}$ and a term $s_{2} \in \mathscr{T}$ such that $\vdash^{*} s_{1}:_{i}(\psi \rightarrow \varphi)$ and $\vdash^{*} s_{2}:_{i} \psi$. So set $t:=s_{1} \cdot \psi s_{2}$ and observe that $\vdash^{*}\left(s_{1} \cdot \psi s_{2}\right):_{i} \varphi$ by Lemma 4.1 and classical propositional reasoning.

While JLCE* does not explicitly include Rule BN, the following lemma shows that this rule is admissible. Since JLCE is just JLCE* plus the rules BN and UN, this lemma gets us one step closer to showing that the theories JLCE* and JLCE derive the same theorems.

Lemma 4.3 (Belief Admissibility) If $\vdash^{*} \varphi$ and $i \in \mathscr{A}$, then $\vdash^{*} B_{i} \varphi$.

Proof Suppose $\vdash^{*} \varphi$ and $i \in \mathscr{A}$. Then for some term $t \in \mathscr{T}$, we have $\vdash^{*} t: i \varphi$ by Lemma 4.2. Since $t:_{i} \varphi$ abbreviates $\left(t \gg_{i} \varphi\right) \wedge B_{i} \varphi$, it follows by classical propositional reasoning that $\vdash^{*} B_{i} \varphi$.

Once we know that Rule BN is admissible, we can show that evidence (and not just relevant evidence) is closed under the term-forming operation !. 

1. $t:_{i} \varphi \rightarrow B_{i} \varphi$
CL
2. $B_{i} \varphi \rightarrow B_{i} B_{i} \varphi$
B2
3. $t:_{i} \varphi \rightarrow t \gg_{i} \varphi$
CL
4. $\left(t \gg_{i} \varphi\right) \rightarrow B_{i}\left(t \gg_{i} \varphi\right)$
E4
5. $t:_{i} \varphi \rightarrow B_{i}\left(t \gg_{i} \varphi\right) \wedge B_{i} B_{i} \varphi$
PR 1-4
6. $B_{i}\left(t:_{i} \varphi\right) \leftrightarrow B_{i}\left(t \gg_{i} \varphi\right) \wedge B_{i} B_{i} \varphi$
MR
7. $t:_{i} \varphi \rightarrow B_{i}\left(t:_{i} \varphi\right)$
PR 5, 6
8. $t \gg_{i} \varphi \rightarrow ! t \gg_{i}\left(t:_{i} \varphi\right)$
E3
9. $t:_{i} \varphi \rightarrow ! t \gg_{i}\left(t:_{i} \varphi\right)$
PR 3, 8
10. $! t:_{i}\left(t:_{i} \varphi\right) \leftrightarrow ! t \gg_{i}\left(t:_{i} \varphi\right) \wedge B_{i}\left(t:_{i} \varphi\right)$
11. $t:_{i} \varphi \rightarrow ! t:_{i}\left(t:_{i} \varphi\right)$
$\mathrm{CL}$
PR 7, 9, 10

Fig. 6 Proof that $\vdash^{*} t:_{i} \varphi \rightarrow ! t:_{i}\left(t:_{i} \varphi\right)$

Lemma 4.4 We have $\vdash^{*} t:_{i} \varphi \rightarrow ! t:_{i}\left(t:_{i} \varphi\right)$.

Proof Using PR to denote the use of classical propositional reasoning and MR to denote the use of modal reasoning (using Lemma 4.3 in place of Rule BN), the proof appears in Fig. 6.

Since Rule BN is admissible in JLCE*, to show that the theories JLCE* and JLCE derive the same theorems, it suffices for us to show that Rule UN is also admissible in JLCE* . Before we begin the proof of this result, we need the following auxiliary lemmas. The first concerns preconditions of a composition and the second concerns associativity of composition.

Lemma 4.5 $\mathrm{p}^{U \circ U^{\prime}}\left(u, u^{\prime}\right) \Leftrightarrow^{*}[U, u] \mathrm{p}^{U^{\prime}}\left(u^{\prime}\right) \wedge \mathrm{p}^{U}(u)$.

Proof Using PR to denote use of classical propositional reasoning:

$$
\begin{aligned}
& \mathrm{p}^{U \circ U^{\prime}}\left(u, u^{\prime}\right) \\
\Leftrightarrow^{*} \neg[U, u] \neg \mathrm{p}^{U^{\prime}}\left(u^{\prime}\right) & \mathrm{CL}, \text { Def. 3.9 } \\
\Leftrightarrow^{*} \neg\left([U, u] \mathrm{p}^{U^{\prime}}\left(u^{\prime}\right) \rightarrow[U, u] \perp\right) & \mathrm{U} 2, \mathrm{PR} \\
\Leftrightarrow^{*} \neg\left([U, u] \mathrm{p}^{U^{\prime}}\left(u^{\prime}\right) \rightarrow\left(\mathrm{p}^{U}(u) \rightarrow \perp\right)\right) & \mathrm{U} 1, \mathrm{PR} \\
\Leftrightarrow^{*}[U, u] \mathrm{p}^{U^{\prime}}\left(u^{\prime}\right) \wedge \mathrm{p}^{U}(u) & \mathrm{PR}
\end{aligned}
$$

Lemma 4.6 $\left[(\bar{U} \circ U) \circ U^{\prime},\left((\bar{u}, u), u^{\prime}\right)\right] \varphi \Leftrightarrow^{*}\left[\bar{U} \circ\left(U \circ U^{\prime}\right),\left(\bar{u},\left(u, u^{\prime}\right)\right)\right] \varphi$.

Proof By induction on the construction of $\varphi .{ }^{9}$ In what follows, update frames will be named using the symbol $U$ perhaps with additional marks such as primes or bars.

\footnotetext{
9 Note that this is actually an induction on $k \in \mathbb{N}$ with a sub-induction on the construction of $\varphi \in \mathscr{F}_{k}$. However, since the sub-inductive arguments are essentially independent of $k$, we shall only present the sub-inductive arguments themselves. It will be apparent from context whether an appeal to the "induction hypothesis" concerns the "inner" sub-induction hypothesis (the statement that the result holds for certain formulas in $\mathscr{F}_{k}$ that have already been constructed) or the "outer" induction hypothesis (the statement that the result holds for formulas in $\bigcup_{i=0}^{k-1} \mathscr{F}_{i}$, all of which have already been constructed).
} 


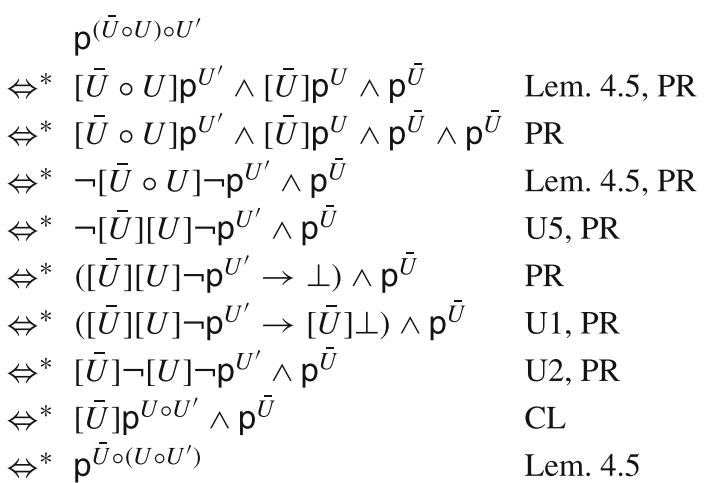

Fig. 7 Proof that $\mathrm{p}^{(\bar{U} \circ U) \circ U^{\prime}} \Leftrightarrow^{*} \mathrm{p}^{\bar{U} \circ\left(U \circ U^{\prime}\right)}$, proof of Lemma 4.6

$$
\begin{aligned}
& {\left[(\bar{U} \circ U) \circ U^{\prime}\right] B_{i} \psi} \\
& \Leftrightarrow{ }^{*} \mathrm{p}^{(\bar{U} \circ U) \circ U^{\prime}} \rightarrow \bigwedge_{u_{\ell} R_{i}^{(\bar{U} \circ U) \circ U^{\prime}} v_{\ell}} B_{i}\left[(\bar{U} \circ U) \circ U^{\prime}, v_{\ell}\right] \psi \quad \mathrm{U} 3
\end{aligned}
$$

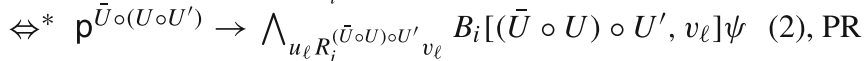

$$
\begin{aligned}
& \Leftrightarrow{ }^{*} \mathrm{p}^{\bar{U} \circ\left(U \circ U^{\prime}\right)} \rightarrow \bigwedge_{u_{\ell} R_{i}^{(\bar{U} \circ U) \circ U^{\prime}} v_{\ell}} B_{i}\left[\bar{U} \circ\left(U \circ U^{\prime}\right), v_{r}\right] \psi \quad \text { IH, MR } \\
& \Leftrightarrow{ }^{*} \mathrm{p}^{\bar{U} \circ\left(U \circ U^{\prime}\right)} \rightarrow \bigwedge_{u_{r} R_{i}^{\bar{U} \circ\left(U \circ U^{\prime}\right)} v_{r}} B_{i}\left[\bar{U} \circ\left(U \circ U^{\prime}\right), v_{r}\right] \psi \text { def'n of } \circ \\
& \Leftrightarrow^{*}\left[\bar{U} \circ\left(U \circ U^{\prime}\right)\right] B_{i} \psi \\
& \text { U3 } \\
& u_{\ell}:=\left((\bar{u}, u), u^{\prime}\right) \quad v_{\ell}:=\left((\bar{v}, v), v^{\prime}\right) \\
& u_{r}:=\left(\bar{u},\left(u, u^{\prime}\right)\right) \quad v_{r}:=\left(\bar{v},\left(v, v^{\prime}\right)\right)
\end{aligned}
$$

Fig. 8 Proof of induction case $B_{i} \psi$, proof of Lemma 4.6

Worlds in update frames will be named using the symbol $u$ with corresponding marks. This allows us to write $\left[U^{\prime}\right]$ to denote the update modal $\left[U^{\prime}, u^{\prime}\right]$, to write $\mathrm{p}^{\bar{U}}$ to denote the formula $\mathrm{p}^{\bar{U}}(\bar{u})$, to write $U \nvdash(t, i, \psi)$ to denote the expression $U, u \nvdash(t, i, \psi)$, to write $\left[U \circ U^{\prime}\right]$ to denote the update modal $\left[U \circ U^{\prime},\left(u, u^{\prime}\right)\right]$, and so on. Proceeding with the proof, many cases require us to prove that

$$
\mathrm{p}^{(\bar{U} \circ U) \circ U^{\prime}} \Leftrightarrow{ }^{*} \mathrm{p}^{\bar{U} \circ\left(U \circ U^{\prime}\right)}
$$

Using PR to denote the use of classical propositional reasoning, we provide the text of the proof for this assertion in Fig. 7 with the understanding that a copy of this text is to be construed as occurring at the beginning of the proofs of those cases where the result (2) is needed. We now provide the proofs of the each case. In what follows, we use IH to denote the use of the induction hypothesis.

- Base (or induction) case $q \in \mathscr{P} \cup\{\perp\}$ is proved using U1, (2) and PR, and U1.

- Induction case $\psi \rightarrow \chi$ with is proved using U2, IH and PR, and U2.

- Induction case $B_{i} \psi$ is proved in Fig. 8; MR denotes the use of modal reasoning (with Lemma 4.3 used in place of Rule BN).

- For induction case $t \gg_{i} \psi$, it follows by Lemma 3.11 that we have the "left" statement $(\bar{U} \circ U) \circ U^{\prime} \vdash(t, i, \psi)$ if and only if $\bar{U} \vdash(t, i, \psi)$ or $U \vdash(t, i, \psi)$ or 


$$
\begin{aligned}
& {\left[(\bar{U} \circ U) \circ U^{\prime}\right][\hat{U}] q} \\
& \Leftrightarrow^{*}\left[\left((\bar{U} \circ U) \circ U^{\prime}\right) \circ \hat{U}\right] q \quad \text { U5 } \\
& \Leftrightarrow \mathrm{p}^{*} \mathrm{p}^{\left((\bar{U} \circ U) \circ U^{\prime}\right) \circ \hat{U}} \rightarrow q \quad \mathrm{U} 1 \\
& \Leftrightarrow^{*}\left(\mathrm{p}^{(\bar{U} \circ U) \circ U^{\prime}} \wedge\left[(\bar{U} \circ U) \circ U^{\prime}\right] \mathrm{p}^{\hat{U}}\right) \rightarrow q \text { Lem. 4.5, PR } \\
& \Leftrightarrow^{*}\left(\mathrm{p}^{\bar{U} \circ\left(U \circ U^{\prime}\right)} \wedge\left[\bar{U} \circ\left(U \circ U^{\prime}\right)\right] \mathrm{p}^{\hat{U}}\right) \rightarrow q \text { (2), IH, PR } \\
& \Leftrightarrow{ }^{*} \mathrm{p}^{\left(\bar{U} \circ\left(U \circ U^{\prime}\right)\right) \circ \hat{U}} \rightarrow q \\
& \Leftrightarrow^{*}\left[\left(\bar{U} \circ\left(U \circ U^{\prime}\right)\right) \circ \hat{U}\right] q \quad \mathrm{U} 1 \\
& \Leftrightarrow^{*}\left[\bar{U} \circ\left(U \circ U^{\prime}\right)\right][\hat{U}] q \quad \text { U5 }
\end{aligned}
$$

Fig. 9 Proof of induction case $[\hat{U}] q$, proof of Lemma 4.6

$U^{\prime} \vdash(t, i, \psi)$. But the latter disjunctive trio is equivalent by Lemma 3.11 to the "right" statement $\bar{U} \circ\left(U \circ U^{\prime}\right) \vdash(t, i, \psi)$. The result is proved using $\mathrm{U} 4$ (with the left statement), (2) and PR, and U4 (with the right statement).

- Induction case $[\hat{U}] q$ with $q \in \mathscr{P} \cup\{\perp\}$ is proved in Fig. 9.

- Induction case $[\hat{U}](\psi \rightarrow \chi)$ is proved using U5, U2, U5 and PR, IH and PR, U5 and PR, U2, and U5.

- Induction case $[\hat{U}] B_{i} \psi$ is proved in Fig. 10.

- For induction case $[\hat{U}]\left(t \gg_{i} \psi\right)$, we observe that, similar to induction case $t \gg_{i} \psi$ above, we have $\left((\bar{U} \circ U) \circ U^{\prime}\right) \circ \hat{U} \vdash(t, i, \psi)$ if and only if $\left(\bar{U} \circ\left(U \circ U^{\prime}\right)\right) \circ \hat{U} \vdash$ $(t, i, \psi)$ by Lemma 3.11. We make use of this biconditional in our proof in Fig. 11 (by assuming that $\mathrm{U} 4$ can be used in the second provable equivalence if and only if $\mathrm{U} 4$ can be used in the second-to-last provable equivalence).

- Induction case $\left[U_{1}\right]\left[U_{2}\right] \psi$ is proved in Fig. 12.

In one part of our proof that Rule UN is JLCE*-admissible, we will show that Rule $\mathrm{UN}$ is $\mathrm{AX}$-admissible. A key case of this proof requires an important lemma about "preconditions" (the forthcoming Lemma 4.7) along with an interesting lemma about substitution (the forthcoming Lemma 4.10).

Lemma 4.7 (Precondition) For each $\varphi \in \mathscr{F}$, we have $[U, u] \varphi \Leftrightarrow{ }^{*} \mathrm{p}^{U}(u) \rightarrow[U, u] \varphi$.

Proof By induction on the construction of $\varphi$, with PR denoting use of classical propositional reasoning and $\mathrm{IH}$ denoting the use of the induction hypothesis.

- $\quad$ Base case $q \in \mathscr{P} \cup\{\perp\}$. See Fig. 13 .

- Induction case $\psi \rightarrow \chi$. See Fig. 14 .

- Induction case $B_{i} \psi$. See Fig. 15.

- Induction case $t \gg_{i} \psi$ is similar to case $B_{i} \psi$, though U4 is used in place of U3. Note that each time U4 is used, the particular form that U4 takes depends on the truth of the single assertion $U, u \vdash(t, i, \psi)$.

- Induction case $\left[U^{\prime}, u^{\prime}\right] \psi$. See Fig. 16.

Definition 4.8 $\left(L(\varphi), S(\varphi)\right.$, Substitutions $\psi \sigma$ and $\left.\psi \sigma^{U, u}\right)$ Let $\varphi \in \mathscr{F} . L(\varphi)$ is the set of propositional letters occurring in $\varphi$ (note that a propositional letter $p_{k}$ occurring 


$$
\begin{aligned}
& {\left[(\bar{U} \circ U) \circ U^{\prime}\right][\hat{U}] B_{i} \psi} \\
& \Leftrightarrow{ }^{*}\left[\left((\bar{U} \circ U) \circ U^{\prime}\right) \circ \hat{U}\right] B_{i} \psi \\
& \Leftrightarrow \mathrm{p}^{\left((\bar{U} \circ U) \circ U^{\prime}\right) \circ \hat{U}} \rightarrow
\end{aligned}
$$

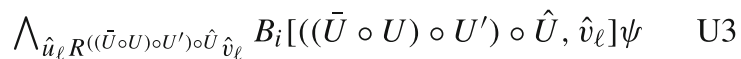

$$
\begin{aligned}
& \Leftrightarrow^{*}\left[(\bar{U} \circ U) \circ U^{\prime}\right] \mathrm{p}^{\hat{U}} \wedge \mathrm{p}^{(\bar{U} \circ U) \circ U^{\prime}} \rightarrow \\
& \bigwedge_{\hat{u}_{\ell} R^{\left((\bar{U} \circ U) \circ U^{\prime}\right) \circ \hat{U}} \hat{v}_{\ell}} B_{i}\left[\left((\bar{U} \circ U) \circ U^{\prime}\right) \circ \hat{U}, \hat{v}_{\ell}\right] \psi \quad \text { Lem. 4.5, PR } \\
& \Leftrightarrow^{*}\left[(\bar{U} \circ U) \circ U^{\prime}\right] \mathrm{p}^{\hat{U}} \wedge \mathrm{p}^{(\bar{U} \circ U) \circ U^{\prime}} \rightarrow
\end{aligned}
$$

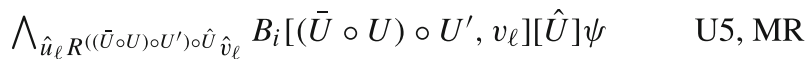

$$
\begin{aligned}
& \Leftrightarrow^{*}\left[\bar{U} \circ\left(U \circ U^{\prime}\right)\right] \mathrm{p}^{\hat{U}} \wedge \mathrm{p}^{\bar{U}\left(\circ U \circ U^{\prime}\right)} \rightarrow \\
& \bigwedge_{\hat{u}_{\ell} R^{\left((\bar{U} \circ U) \circ U^{\prime}\right) \circ \hat{U}} \hat{v}_{\ell}} B_{i}\left[(\bar{U} \circ U) \circ U^{\prime}, v_{\ell}\right][\hat{U}] \psi \quad \mathrm{IH},(2), \mathrm{PR} \\
& \Leftrightarrow^{*}\left[\bar{U} \circ\left(U \circ U^{\prime}\right)\right] \mathrm{p}^{\hat{U}} \wedge \mathrm{p}^{\bar{U}\left(\circ U \circ U^{\prime}\right)} \rightarrow
\end{aligned}
$$

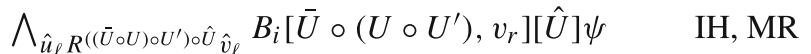

$$
\begin{aligned}
& \Leftrightarrow^{*}\left[\bar{U} \circ\left(U \circ U^{\prime}\right)\right] \mathrm{p}^{\hat{U}} \wedge \mathrm{p}^{\bar{U}\left(\circ U \circ U^{\prime}\right)} \rightarrow \\
& \bigwedge_{\hat{u}_{\ell} R^{\left((\bar{U} \circ U) \circ U^{\prime}\right) \circ \hat{U}} \hat{v}_{\ell}} B_{i}\left[\left(\bar{U} \circ\left(U \circ U^{\prime}\right)\right) \circ \hat{U}, \hat{v}_{r}\right] \psi \quad \mathrm{U} 5, \mathrm{MR} \\
& \Leftrightarrow^{*} \mathrm{p}^{\left(\bar{U} \circ\left(U \circ U^{\prime}\right)\right) \circ \hat{U}} \rightarrow \\
& \bigwedge_{\hat{u}_{\ell} R^{\left((\bar{U} \circ U) \circ U^{\prime}\right) \circ \hat{U}} \hat{v}_{\ell}} B_{i}\left[\left(\bar{U} \circ\left(U \circ U^{\prime}\right)\right) \circ \hat{U}, \hat{v}_{r}\right] \psi \quad \text { Lem. 4.5, PR } \\
& \Leftrightarrow^{*} \mathrm{p}^{\left(\bar{U} \circ\left(U \circ U^{\prime}\right)\right) \circ \hat{U}} \rightarrow \\
& \bigwedge_{\hat{u}_{r} R^{\left(\bar{U} \circ\left(U \circ U^{\prime}\right)\right) \circ \hat{U}} \hat{v}_{r}} B_{i}\left[\left(\bar{U} \circ\left(U \circ U^{\prime}\right)\right) \circ \hat{U}, \hat{v}_{r}\right] \psi \quad \operatorname{def} \text { 'n of } \circ \\
& \Leftrightarrow{ }^{*}\left[\left(\bar{U} \circ\left(U \circ U^{\prime}\right)\right) \circ \hat{U}\right] B_{i} \psi \\
& \Leftrightarrow^{*}\left[\bar{U} \circ\left(U \circ U^{\prime}\right)\right][\hat{U}] B_{i} \psi \\
& \hat{u}_{\ell}:=\left(\left((\bar{u}, u), u^{\prime}\right), \hat{u}\right) \quad \hat{v}_{\ell}:=\left(\left((\bar{v}, v), v^{\prime}\right), \hat{v}\right) \\
& \hat{u}_{r}:=\left(\left(\bar{u},\left(u, u^{\prime}\right)\right), \hat{u}\right) \quad v_{\ell}:=\left((\bar{v}, v), v^{\prime}\right) \\
& v_{r}:=\left(\bar{v},\left(v, v^{\prime}\right)\right) \\
& \hat{v}_{r}:=\left(\left(\bar{v},\left(v, v^{\prime}\right)\right), \hat{v}\right)
\end{aligned}
$$

Fig. 10 Proof of induction case $[\hat{U}] B_{i} \psi$, proof of Lemma 4.6

$$
\begin{aligned}
& {\left[(\bar{U} \circ U) \circ U^{\prime}\right][\hat{U}] t \gg_{i} \psi} \\
& \Leftrightarrow^{*}\left[\left((\bar{U} \circ U) \circ U^{\prime}\right) \circ \hat{U}\right] t \gg_{i} \psi \quad \text { U5 } \\
& \Leftrightarrow \mathrm{p}^{\left((\bar{U} \circ U) \circ U^{\prime}\right) \circ \hat{U}} \rightarrow X \quad \mathrm{U} 4 \\
& \Leftrightarrow^{*}\left[(\bar{U} \circ U) \circ U^{\prime}\right] \mathrm{p}^{\hat{U}} \wedge \mathrm{p}^{(\bar{U} \circ U) \circ U^{\prime}} \rightarrow X \text { Lem. 4.5, PR } \\
& \Leftrightarrow^{*}\left[\bar{U} \circ\left(U \circ U^{\prime}\right)\right] \mathrm{p}^{\hat{U}} \wedge \mathrm{p}^{\bar{U} \circ\left(U \circ U^{\prime}\right)} \rightarrow X \quad \mathrm{IH},(2), \mathrm{PR} \\
& \Leftrightarrow^{*} \mathrm{p}^{\left(\bar{U} \circ\left(U \circ U^{\prime}\right)\right) \circ \hat{U}} \rightarrow X \\
& \Leftrightarrow^{*}\left[\left(\bar{U} \circ\left(U \circ U^{\prime}\right)\right) \circ \hat{U}\right] t \gg_{i} \psi \quad \mathrm{U} 4 \\
& \Leftrightarrow^{*}\left[\bar{U} \circ\left(U \circ U^{\prime}\right)\right][\hat{U}] t \gg_{i} \psi \quad \mathrm{U} 5 \\
& X=\perp \text { or } X=t \gg_{i} \psi \\
& \text { Lem. 4.5, PR } \\
& \text { U4 }
\end{aligned}
$$

Fig. 11 Proof of induction case $[\hat{U}] t \gg_{i} \psi$, proof of Lemma 4.6

in $\varphi$ may occur within an update modal $[U, u]$ that itself occurs in $\varphi) . S(\varphi)$ is the set of functions $\sigma: L(\varphi) \rightarrow \mathscr{F}$ that map the natural-number subscript $k$ of propositional 


$$
\begin{aligned}
& {\left[(\bar{U} \circ U) \circ U^{\prime}\right]\left[U_{1}\right]\left[U_{2}\right] \psi} \\
& \Leftrightarrow^{*}\left[\left(\left((\bar{U} \circ U) \circ U^{\prime}\right) \circ U_{1}\right) \circ U_{2}\right] \psi \mathrm{U} 5 \\
& \Leftrightarrow^{*}\left[\left((\bar{U} \circ U) \circ U^{\prime}\right) \circ\left(U_{1} \circ U_{2}\right)\right] \psi \mathrm{IH} \\
& \Leftrightarrow^{*}\left[(\bar{U} \circ U) \circ U^{\prime}\right]\left[U_{1} \circ U_{2}\right] \psi \quad \mathrm{U} 5 \\
& \Leftrightarrow^{*}\left[\bar{U} \circ\left(U \circ U^{\prime}\right)\right]\left[U_{1} \circ U_{2}\right] \psi \quad \mathrm{IH} \\
& \Leftrightarrow^{*}\left[\left(\bar{U} \circ\left(U \circ U^{\prime}\right)\right) \circ\left(U_{1} \circ U_{2}\right)\right] \psi \mathrm{U} 5 \\
& \Leftrightarrow^{*}\left[\left(\left(\bar{U} \circ\left(U \circ U^{\prime}\right)\right) \circ U_{1}\right) \circ U_{2}\right] \psi \mathrm{IH} \\
& \Leftrightarrow^{*}\left[\bar{U} \circ\left(U \circ U^{\prime}\right)\right]\left[U_{1}\right]\left[U_{2}\right] \psi \quad \text { U5 }
\end{aligned}
$$

Fig. 12 Proof of induction case $\left[U_{1}\right]\left[U_{2}\right] \psi$, proof of Lemma 4.6

$$
\begin{array}{rlrl}
{[U, u] q} & \Leftrightarrow \mathrm{p}^{U}(u) \rightarrow q & \mathrm{U} 1 \\
& \Leftrightarrow \mathrm{p}^{U}(u) \rightarrow\left(\mathrm{p}^{U}(u) \rightarrow q\right) & \mathrm{PR} \\
& \Leftrightarrow * \mathrm{p}^{U}(u) \rightarrow[U, u] q & \mathrm{U} 1
\end{array}
$$

Fig. 13 Base case $q \in \mathscr{P} \cup\{\perp\}$, Lemma 4.7

$$
\begin{aligned}
& {[U, u](\psi \rightarrow \chi)} \\
& \Leftrightarrow^{*}[U, u] \psi \rightarrow[U, u] \chi \\
& \Leftrightarrow^{*}\left(\mathrm{p}^{U}(u) \rightarrow[U, u] \psi\right) \rightarrow\left(\mathrm{p}^{U}(u) \rightarrow[U, u] \chi\right) \text { IH, PR } \\
& \Leftrightarrow \mathrm{p}^{U}(u) \rightarrow([U, u] \psi \rightarrow[U, u] \chi) \quad \mathrm{PR} \\
& \Leftrightarrow^{*} \mathrm{p}^{U}(u) \rightarrow[U, u](\psi \rightarrow \chi) \quad \text { U2, PR }
\end{aligned}
$$

Fig. 14 Induction case $\psi \rightarrow \chi$, Lemma 4.7

$$
\begin{array}{rlr} 
& {[U, u] B_{i} \psi} \\
\Leftrightarrow & { }^{*} \mathrm{p}^{U}(u) \rightarrow \bigwedge_{u R_{i}^{U} v} B_{i}[U, v] \psi & \mathrm{U} 3 \\
\Leftrightarrow & *^{*} \mathrm{p}^{U}(u) \rightarrow\left(\mathrm{p}^{U}(u) \rightarrow \bigwedge_{u R_{i}^{U} v} B_{i}[U, v] \psi\right) & \mathrm{PR} \\
\Leftrightarrow & { }^{*} \mathrm{p}^{U}(u) \rightarrow[U, u] B_{i} \psi & \mathrm{U} 3,
\end{array}
$$

Fig. 15 Induction case $B_{i} \psi$, Lemma 4.7

letter $p_{k}$ occurring in $\varphi$ to formula $\sigma(k) \in \mathscr{F}$. To define substitution of formulas for propositional letters: given $\sigma \in S(\varphi)$ and $\psi \in \mathscr{F}$, we let $\psi \sigma$ denote the formula obtained from $\psi$ by simultaneously replacing for each $k \in L(\varphi)$ all occurrences of the propositional letter $p_{k}$ in $\psi$ by the formula $\sigma(k)$. Note that for $p_{k}$ to be replaced in $\psi$ by a substitution $\sigma \in S(\varphi)$, the propositional letter $p_{k}$ must occur both in $\psi$

$$
\begin{aligned}
& {[U, u]\left[U^{\prime}, u^{\prime}\right] \psi} \\
& \Leftrightarrow^{*}\left[U \circ U^{\prime},\left(u, u^{\prime}\right)\right] \psi \quad \mathrm{U} 5 \\
& \Leftrightarrow^{*} \mathrm{p}^{U \circ U^{\prime}}\left(u, u^{\prime}\right) \rightarrow\left[U \circ U^{\prime},\left(u, u^{\prime}\right)\right] \psi \quad \mathrm{IH} \\
& \Leftrightarrow{ }^{*} \mathrm{p}^{U}(u) \wedge[U, u] \mathrm{p}^{U^{\prime}}\left(u^{\prime}\right) \rightarrow\left[U \circ U^{\prime},\left(u, u^{\prime}\right)\right] \psi \quad \text { Lem. 4.5, PR } \\
& \Leftrightarrow^{*} \mathrm{p}^{U}(u) \rightarrow\left(\mathrm{p}^{U}(u) \wedge[U, u] \mathrm{p}^{U^{\prime}}\left(u^{\prime}\right) \rightarrow\left[U \circ U^{\prime},\left(u, u^{\prime}\right)\right] \psi\right) \text { PR } \\
& \Leftrightarrow^{*} \mathrm{p}^{U}(u) \rightarrow\left(\mathrm{p}^{U \circ U^{\prime}}\left(u, u^{\prime}\right) \rightarrow\left[U \circ U^{\prime},\left(u, u^{\prime}\right)\right] \psi\right) \quad \text { Lem. 4.5, PR } \\
& \Leftrightarrow^{*} \mathrm{p}^{U}(u) \rightarrow\left[U \circ U^{\prime},\left(u, u^{\prime}\right)\right] \psi \quad \text { IH, PR } \\
& \Leftrightarrow^{*} \mathrm{p}^{U}(u) \rightarrow[U, u]\left[U^{\prime}, u^{\prime}\right] \psi \quad \text { U5, PR }
\end{aligned}
$$

Fig. 16 Induction case $\left[U^{\prime}, u^{\prime}\right] \psi$, Lemma 4.7 


$$
\begin{aligned}
& {[U, u]\left(\left(\psi_{1} \rightarrow \psi_{2}\right) \sigma\right)} \\
& \Leftrightarrow^{*}[U, u]\left(\psi_{1} \sigma \rightarrow \psi_{2} \sigma\right) \\
& \Leftrightarrow{ }^{*}[U, u]\left(\psi_{1} \sigma\right) \rightarrow[U, u]\left(\psi_{2} \sigma\right) \\
& \Leftrightarrow^{*}\left(\mathrm{p}^{U}(u) \rightarrow\left(\psi_{1} \sigma^{U, u}\right)\right) \rightarrow\left(\mathrm{p}^{U}(u) \rightarrow\left(\psi_{2} \sigma^{U, u}\right)\right) \text { IH, PR } \\
& \Leftrightarrow^{*} \mathrm{p}^{U}(u) \rightarrow\left(\psi_{1} \sigma^{U, u} \rightarrow \psi_{2} \sigma^{U, u}\right) \\
& \text { PR } \\
& \Leftrightarrow{ }^{*} \mathrm{p}^{U}(u) \rightarrow\left(\left(\psi_{1} \rightarrow \psi_{2}\right) \sigma^{U, u}\right) \\
& \text { def'n of substitution }
\end{aligned}
$$

Fig. 17 Induction case $\psi_{1} \rightarrow \psi_{2}$, Lemma 4.10

and in $\varphi$. Given $(U, u) \in \mathscr{U}, \varphi \in \mathscr{F}$, and $\sigma \in S(\varphi)$, we define the substitution $\sigma^{U, u}: L(\varphi) \rightarrow \mathscr{F}$ by setting $\sigma^{U, u}(k):=[U, u] \sigma(k)$ for each $k \in L(\varphi)$.

Definition 4.9 (Propositional Logic PL) PL is the set of formulas in the language of propositional logic having atoms $q \in \mathscr{P} \cup\{\perp\}$ and the binary Boolean connective $\rightarrow$ for implication. Use of logical connectives not in this language is to be understood as an abbreviation of the expression that uses connectives that do appear in this language. We observe that PL is a sublanguage of UL.

Lemma 4.10 (Substitution) For each $\psi \in \mathrm{PL}$, each $\sigma \in S(\psi)$, and each $(U, u) \in \mathscr{U}$, we have $[U, u](\psi \sigma) \Leftrightarrow{ }^{*} \mathrm{p}^{U}(u) \rightarrow\left(\psi \sigma^{U, u}\right)$.

Proof By induction on the construction of $\psi \in \mathrm{PL}$, with IH denoting use of the induction hypothesis and PR denoting use of classical propositional reasoning.

- Base case: $\psi=p \in \mathscr{P}$. We have $[U, u](p \sigma) \Leftrightarrow{ }^{*} \mathrm{p}^{U}(u) \rightarrow[U, u](p \sigma)$ by Lemma 4.7, and we have $[U, u](p \sigma)=p \sigma^{U, u}$ by the definition of $\sigma^{U, u}$.

- Base case: $\psi=\perp$. We have $\perp \sigma=\perp$ by the definition of substitution, and we have $[U, u] \perp \Leftrightarrow^{*} \mathrm{p}^{U}(u) \rightarrow \perp$ by Axiom U1.

- Induction case: $\psi=\psi_{1} \rightarrow \psi_{2}$. See Fig. 17.

We are now ready to prove the JLCE* -admissibility of Rule UN. We begin by showing that this rule is $A X$-admissible.

Lemma 4.11 (AX Update Admissibility) $\mathrm{AX} \vdash \varphi$ implies $\vdash^{*}[\bar{U}, \bar{u}] \varphi$.

Proof By induction on the length of AX derivations, using PR to denote the use of classical propositional reasoning and MR to denote the use of modal reasoning (using Lemma 4.3 in place of rule $\mathrm{BN}$ ).

- Base case: Axiom CL. Given an instance $\varphi \in \mathscr{F}$ of CL, there exists a classical propositional tautology $\psi \in \mathrm{PL}$ and a substitution $\sigma \in S(\psi)$ such that $\varphi=\psi \sigma$. Choose an arbitrary $(\bar{U}, \bar{u}) \in \mathscr{U}$. Since $\psi$ is a tautology, it follows that $\vdash^{*} \psi \sigma^{\bar{U}, \bar{u}}$ by Axiom CL and hence that $\vdash^{*} \mathrm{p}^{\bar{U}}(\bar{u}) \rightarrow\left(\psi \sigma^{\bar{U}, \bar{u}}\right)$ by PR. Applying Lemma 4.10, it follows that $\vdash^{*}[\bar{U}, \bar{u}](\psi \sigma)$. Since $\varphi=\psi \sigma$, we have shown that $\vdash^{*}[\bar{U}, \bar{u}] \varphi$, as desired.

- Base case: Axiom B1. See Fig. 18.

- Base case: Axiom B2. See Fig. 19.

- Base case: Axiom E1. For each $t \in \mathscr{T}, i \in \mathscr{A}, \psi \in \mathscr{F}$, and $(U, u) \in \mathscr{U}$, define the formula $(t, i, \psi)^{U, u}$ by setting 
1. $\bigwedge_{\bar{u} R_{i}^{\bar{U}} \bar{v}}([\bar{U}, \bar{v}] \varphi \rightarrow[\bar{U}, \bar{v}] \psi) \rightarrow$

$$
\left(\bigwedge_{\bar{u} R_{i}^{\bar{U}} \bar{v}}[\bar{U}, \bar{v}] \varphi \rightarrow \bigwedge_{\bar{u} R_{i}^{\bar{U}} \bar{v}}[\bar{U}, \bar{v}] \psi\right) \quad \text { PR }
$$

2. $\bigwedge_{\bar{u} R_{i}^{\bar{U}} \bar{v}}[\bar{U}, \bar{v}](\varphi \rightarrow \psi) \rightarrow$

$$
\left(\bigwedge_{\bar{u} R_{i}^{\bar{U}} \bar{v}}[\bar{U}, \bar{v}] \varphi \rightarrow \bigwedge_{\bar{u} R_{i}^{\bar{U}} \bar{v}}[\bar{U}, \bar{v}] \psi\right) \quad \mathrm{U} 2, \mathrm{PR} 1
$$

3. $\bigwedge_{\bar{u} R_{i}^{\bar{U}} \bar{v}} B_{i}[\bar{U}, \bar{v}](\varphi \rightarrow \psi) \rightarrow$

$$
\left(\bigwedge_{\bar{u} R_{i}^{\bar{U}} \bar{v}} B_{i}[\bar{U}, \bar{v}] \varphi \rightarrow \bigwedge_{\bar{u} R_{i}^{\bar{U}} \bar{v}} B_{i}[\bar{U}, \bar{v}] \psi\right) \quad \text { MR } 2
$$

4. $\left(\mathrm{p}^{\bar{U}}(\bar{u}) \rightarrow \bigwedge_{\bar{u} R_{i}^{\bar{U}} \bar{v}} B_{i}[\bar{U}, \bar{v}](\varphi \rightarrow \psi)\right) \rightarrow$

$$
\begin{gathered}
\left(\left(\mathrm{p}^{\bar{U}}(\bar{u}) \rightarrow \bigwedge_{\bar{u} R_{i}^{\bar{U}} \bar{v}} B_{i}[\bar{U}, \bar{v}] \varphi\right) \rightarrow\right. \\
\left.\left(\mathrm{p}^{\bar{U}}(\bar{u}) \rightarrow \bigwedge_{\bar{u} R_{i}^{\bar{U}} \bar{v}} B_{i}[\bar{U}, \bar{v}] \psi\right)\right)
\end{gathered}
$$

5. $[\bar{U}, \bar{u}] B_{i}(\varphi \rightarrow \psi) \rightarrow\left([\bar{U}, \bar{u}] B_{i} \varphi \rightarrow[\bar{U}, \bar{u}] B_{i} \psi\right) \quad \mathrm{U} 3, \mathrm{MR} 4$

6. $[\bar{U}, \bar{u}]\left(B_{i}(\varphi \rightarrow \psi) \rightarrow\left(B_{i} \varphi \rightarrow B_{i} \psi\right)\right) \quad$ U2, PR 5

Fig. 18 Base case B1, proof of Lemma 4.11
1. $\quad B_{i}\left(\bigwedge_{\bar{u} R_{i}^{\bar{U}} \bar{v}}[\bar{U}, \bar{v}] \varphi\right) \rightarrow B_{i} B_{i}\left(\bigwedge_{\bar{u} R_{i}^{\bar{U}} \bar{v}}[\bar{U}, \bar{v}] \varphi\right)$
B2
2. $B_{i}\left(\bigwedge_{\bar{u} R_{i}^{\bar{U}} \bar{v}}[\bar{U}, \bar{v}] \varphi\right) \rightarrow B_{i}\left(\bigwedge_{\bar{u} R_{i}^{\bar{U}} \bar{v}} B_{i}[\bar{U}, \bar{v}] \varphi\right)$
MR 1
3. $\left(\bigwedge_{\bar{u} R_{i}^{\bar{U}} \bar{v}}[\bar{U}, \bar{v}] \varphi\right) \rightarrow \bigwedge_{\bar{u} R_{i}^{\bar{U}} \bar{v}}\left(\bigwedge_{\bar{v} R_{i}^{\bar{U}} \bar{w}}[\bar{U}, \bar{w}] \varphi\right)$ $R_{i}^{\bar{U}}$ trans.
4. $\quad\left(\bigwedge_{\bar{u} R_{i}^{\bar{U}} \bar{v}} B_{i}[\bar{U}, \bar{v}] \varphi\right) \rightarrow \bigwedge_{\bar{u} R_{i}^{\bar{U}} \bar{v}}\left(\bigwedge_{\bar{v} R_{i}^{\bar{U}} \bar{w}} B_{i}[\bar{U}, \bar{w}] \varphi\right)$
MR 3
5. $\quad\left(\bigwedge_{\bar{u} R_{i}^{\bar{U}} \bar{v}} B_{i}[\bar{U}, \bar{v}] \varphi\right) \rightarrow \bigwedge_{\bar{u} R_{i}^{\bar{U}} \bar{v}}\left(\mathrm{p}^{\bar{U}}(\bar{v}) \rightarrow \bigwedge_{\bar{v} R_{i}^{\bar{U}} \bar{w}} B_{i}[\bar{U}, \bar{w}] \varphi\right) \quad \operatorname{PR} 4$
6. $B_{i}\left(\bigwedge_{\bar{u} R_{i}^{\bar{U}} \bar{v}} B_{i}[\bar{U}, \bar{v}] \varphi\right) \rightarrow$

$$
\bigwedge_{\bar{u} R_{i}^{\bar{U}} \bar{v}} B_{i}\left(\mathrm{p}^{\bar{U}}(\bar{v}) \rightarrow \bigwedge_{\bar{v} R_{i}^{\bar{U}} \bar{w}} B_{i}[\bar{U}, \bar{w}] \varphi\right)
$$
MR 5
7. $B_{i}\left(\bigwedge_{\bar{u} R_{i}^{\bar{U}} \bar{v}}[\bar{U}, \bar{v}] \varphi\right) \rightarrow$

$$
\bigwedge_{\bar{u} R_{i}^{\bar{U}} \bar{v}} B_{i}\left(\mathrm{p}^{\bar{U}}(\bar{v}) \rightarrow \bigwedge_{\bar{v} R_{i}^{\bar{U}} \bar{w}} B_{i}[\bar{U}, \bar{w}] \varphi\right)
$$
8. $\quad B_{i}\left(\bigwedge_{\bar{u} R_{i}^{\bar{U}} \bar{v}}[\bar{U}, \bar{v}] \varphi\right) \rightarrow \bigwedge_{\bar{u} R_{i}^{\bar{U}} \bar{v}} B_{i}[\bar{U}, \bar{v}] B_{i} \varphi$
9. $\left(\bigwedge_{\bar{u} R_{i}^{\bar{U}} \bar{v}} B_{i}[\bar{U}, \bar{v}] \varphi\right) \rightarrow \bigwedge_{\bar{u} R_{i}^{\bar{U}} \bar{v}} B_{i}[\bar{U}, \bar{v}] B_{i} \varphi$
MR 8
10. $\left(\mathrm{p}^{\bar{U}}(\bar{u}) \rightarrow \bigwedge_{\bar{u} R_{i}^{\bar{U}} \bar{v}} B_{i}[\bar{U}, \bar{v}] \varphi\right) \rightarrow$

$$
\left(\mathrm{p}^{\bar{U}}(\bar{u}) \rightarrow \bigwedge_{\bar{u} R_{i}^{\bar{U}} \bar{v}} B_{i}[\bar{U}, \bar{v}] B_{i} \varphi\right)
$$
11. $[\bar{U}, \bar{u}] B_{i} \varphi \rightarrow[\bar{U}, \bar{u}] B_{i} B_{i} \varphi$
12. $[\bar{U}, \bar{u}]\left(B_{i} \varphi \rightarrow B_{i} B_{i} \varphi\right)$

Fig. 19 Base case B2, proof of Lemma 4.11

$$
(t, i, \psi)^{U, u}:= \begin{cases}\neg \mathrm{p}^{U}(u) & \text { if } U, u \vdash(t, i, \psi) \\ \mathrm{p}^{U}(u) \rightarrow t \gg_{i} \psi & \text { if } U, u \nvdash(t, i, \psi) .\end{cases}
$$

We let $(t, i, \psi)_{1}^{U, u}$ mean that $(t, i, \psi)^{U, u}=\neg \mathrm{p}^{U}(u)$, and $(t, i, \psi)_{0}^{U, u}$ denote the negation of $(t, i, \psi)_{1}^{U, u}$. By inspection of the $\bar{U}$-calculus (Fig. 1), it is clear that we have 


$$
\left((t, i, \varphi \rightarrow \psi)_{1}^{\bar{U}, \bar{u}} \quad \text { or }(s, i, \varphi)_{1}^{\bar{U}, \bar{u}}\right) \quad \text { iff }(t \cdot \varphi s, i, \psi)_{1}^{\bar{U}, \bar{u}}
$$

Further, we have the following chain of provable equivalences.

$$
\begin{aligned}
& {[\bar{U}, \bar{u}]\left(t \gg_{i}(\varphi \rightarrow \psi) \rightarrow\left(s \gg_{i} \varphi \rightarrow t \cdot_{\varphi} s \gg_{i} \psi\right)\right) } \\
\Leftrightarrow^{*}[\bar{U}, \bar{u}]\left(t \gg_{i}(\varphi \rightarrow \psi)\right) \rightarrow\left([\bar{U}, \bar{u}]\left(s \gg_{i} \varphi\right) \rightarrow[\bar{U}, \bar{u}]\left(t \cdot \varphi s \gg_{i} \psi\right)\right) & \mathrm{U} 2, \mathrm{PR} \\
\Leftrightarrow^{*}(t, i, \varphi \rightarrow \psi)^{\bar{U}, \bar{u}} \rightarrow\left((s, i, \varphi)^{\bar{U}, \bar{u}} \rightarrow(t \cdot \varphi s, i, \psi)^{\bar{U}, \bar{u}}\right) & \mathrm{U} 4, \mathrm{PR}
\end{aligned}
$$

If $(t \cdot \varphi s, i, \psi)_{1}^{\bar{U}, \bar{u}}$, then it follows by (3) that $(t, i, \varphi \rightarrow \psi)_{1}^{\bar{U}, \bar{u}}$ or $(s, i, \varphi)_{1}^{\bar{U}, \bar{u}}$ and hence that the last formula in the above chain of provable equivalences is an instance of Axiom CL. If $(t \cdot \varphi s, i, \psi)_{0}^{\bar{U}, \bar{u}}$, then it follows by (3) that $(t, i, \varphi \rightarrow$ $\psi)_{0}^{\bar{U}, \bar{u}}$ and $(s, i, \varphi)_{0}^{\bar{U}, \bar{u}}$; the last formula in the above chain is therefore $\mathrm{JLCE}^{*}$ provably equivalent (by PR) to

$$
\mathrm{p}^{\bar{U}}(\bar{u}) \rightarrow\left(t \gg_{i}(\varphi \rightarrow \psi) \rightarrow\left(\left(s \gg_{i} \varphi\right) \rightarrow\left(t \cdot \varphi s \gg_{i} \psi\right)\right)\right)
$$

which is itself JLCE*-provable by PR from Axiom E1.

- Base case: Axiom E2. By inspection of the $U$-calculus (Fig. 1), it is clear that we have

$$
\left((t, i, \varphi)_{1}^{\bar{U}, \bar{u}} \quad \text { and } \quad(s, i, \varphi)_{1}^{\bar{U}, \bar{u}}\right) \quad \text { iff }(t+s, i, \varphi)_{1}^{\bar{U}, \bar{u}}
$$

Further, we have the following chain of provable equivalences.

$$
\begin{aligned}
& {[\bar{U}, \bar{u}]\left(t \gg_{i} \varphi \rightarrow t+s \gg_{i} \varphi\right)} \\
& \Leftrightarrow^{*}[\bar{U}, \bar{u}]\left(t \gg_{i} \varphi\right) \rightarrow[\bar{U}, \bar{u}]\left(t+s \gg_{i} \varphi\right) \quad \mathrm{U} 2 \\
& \Leftrightarrow{ }^{*}(t, i, \varphi)^{\bar{U}, \bar{u}} \rightarrow(t+s, i, \varphi)^{\bar{U}, \bar{u}} \quad \mathrm{U} 4, \mathrm{PR}
\end{aligned}
$$

If $(t+s, i, \varphi)_{1}^{\bar{U}, \bar{u}}$, then it follows by (4) that $(t, i, \varphi)_{1}^{\bar{U}, \bar{u}}$ and hence that the last formula in the above chain of provable equivalences is an instance of Axiom CL. If $(t+s, i, \varphi)_{0}^{\bar{U}, \bar{u}}$, then it follows by (4) that $(t, i, \varphi)_{0}^{\bar{U}, \bar{u}}$ and hence that the last formula in the above chain is JLCE* -provably equivalent to

$$
\mathrm{p}^{\bar{U}}(\bar{u}) \rightarrow\left(\left(t \gg_{i} \varphi\right) \rightarrow\left(t+s \gg_{i} \varphi\right)\right)
$$

which is itself JLCE* -provable by PR from Axiom E2. The argument for the other form of Axiom E2, $\left(s \gg_{i} \varphi\right) \rightarrow\left(t+s \gg_{i} \varphi\right)$, is similar.

- Base case: Axiom E3. The argument is similar to the argument for Axiom E2, though Axiom E3 is used at the end in place of Axiom E2. 


$$
\begin{aligned}
& {[\bar{U}, \bar{u}][U, u] q} \\
& \Leftrightarrow^{*}[\bar{U} \circ U,(\bar{u}, u)] q \quad \text { U5 } \\
& \Leftrightarrow \mathrm{p}^{*} \circ \mathrm{U}^{\bar{U}}(\bar{u}, u) \rightarrow q \quad \mathrm{U} 1 \\
& \Leftrightarrow \Leftrightarrow^{*}\left(\mathrm{p}^{\bar{U}}(\bar{u}) \wedge[\bar{U}, \bar{u}] \mathrm{p}^{U}(u)\right) \rightarrow q \quad \text { Lem. 4.5, PR } \\
& \Leftrightarrow{ }^{*}[\bar{U}, \bar{u}] \mathrm{p}^{U}(u) \rightarrow\left(\mathrm{p}^{\bar{U}}(\bar{u}) \rightarrow q\right) \text { PR } \\
& \Leftrightarrow{ }^{*}[\bar{U}, \bar{u}] \mathrm{p}^{U}(u) \rightarrow[\bar{U}, \bar{u}] q \quad \mathrm{U} 1, \mathrm{PR} \\
& \Leftrightarrow{ }^{*}[\bar{U}, \bar{u}]\left(\mathrm{p}^{U}(u) \rightarrow q\right) \quad \mathrm{U} 2
\end{aligned}
$$

Fig. 20 Base case U1, proof of Lemma 4.11

$$
\begin{aligned}
& {[\bar{U}, \bar{u}][U, u](\varphi \rightarrow \psi)} \\
& \Leftrightarrow^{*}[\bar{U} \circ U,(\bar{u}, u)](\varphi \rightarrow \psi) \quad \text { U5 } \\
& \Leftrightarrow^{*}[\bar{U} \circ U,(\bar{u}, u)] \varphi \rightarrow[\bar{U} \circ U,(\bar{u}, u)] \psi \quad \mathrm{U} 2 \\
& \Leftrightarrow^{*}[\bar{U}, \bar{u}][U, u] \varphi \rightarrow[\bar{U}, \bar{u}][U, u] \psi \quad \text { U5, PR } \\
& \Leftrightarrow^{*}[\bar{U}, \bar{u}]([U, u] \varphi \rightarrow[U, u] \psi) \quad \mathrm{U} 2
\end{aligned}
$$

Fig. 21 Base case U2, proof of Lemma 4.11

- Base case: Axiom E4. Consider the following chain of provable equivalences.

$$
\begin{array}{rlr} 
& {[\bar{U}, \bar{u}]\left(t \gg_{i} \varphi \rightarrow B_{i}\left(t \gg_{i} \varphi\right)\right)} & \\
\Leftrightarrow & { }^{*}[\bar{U}, \bar{u}]\left(t \gg_{i} \varphi\right) \rightarrow[\bar{U}, \bar{u}] B_{i}\left(t \gg_{i} \varphi\right) & \mathrm{U} 2 \\
\Leftrightarrow & *^{*}(t, i, \varphi)^{\bar{U}, \bar{u}} \rightarrow[\bar{U}, \bar{u}] B_{i}\left(t \gg_{i} \varphi\right) & \mathrm{U} 4, \mathrm{PR} \\
\Leftrightarrow & { }^{*}(t, i, \varphi)^{\bar{U}, \bar{u}} \rightarrow\left(\mathrm{p}^{\bar{U}}(\bar{u}) \rightarrow \bigwedge_{\bar{u} R_{i}^{\bar{U}} \bar{v}} B_{i}[\bar{U}, \bar{v}]\left(t \gg_{i} \varphi\right)\right) & \mathrm{U} 3, \mathrm{PR} \\
\Leftrightarrow & { }^{*}(t, i, \varphi)^{\bar{U}, \bar{u}} \rightarrow\left(\mathrm{p}^{\bar{U}}(\bar{u}) \rightarrow \bigwedge_{\bar{u} R_{i}^{\bar{U}} \bar{v}} B_{i}(t, i, \varphi)^{\bar{U}, \bar{v}}\right) & \mathrm{U} 4, \mathrm{MR}
\end{array}
$$

If $(t, i, \varphi)_{1}^{\bar{U}, \bar{u}}$, then the last line of the above chain of provable equivalences is an instance of Axiom CL. So let us assume that $(t, i, \varphi)_{0}^{\bar{U}, \bar{u}}$ and hence that $(t, i, \varphi)_{0}^{\bar{U}, \bar{u}}$ by Lemma 3.7. The following is then a JLCE* -derivation of the last line of the above chain.

$$
\begin{aligned}
& \text { 1. }\left(t \gg_{i} \varphi\right) \rightarrow B_{i}\left(t \gg_{i} \varphi\right) \\
& \text { E4 } \\
& \text { 2. }\left(t \gg_{i} \varphi\right) \rightarrow \bigwedge_{\bar{u} R_{i}^{\bar{U}} \bar{v}} B_{i}\left(\mathrm{p}^{\bar{U}}(\bar{v}) \rightarrow t \gg_{i} \varphi\right) \quad \text { MR } 1 \\
& \text { 3. }\left(\mathrm{p}^{\bar{U}}(\bar{u}) \rightarrow t \gg_{i} \varphi\right) \rightarrow \\
& \left(\mathrm{p}^{\bar{U}}(\bar{u}) \rightarrow \bigwedge_{\bar{u} R_{i}^{\bar{U}} \bar{v}} B_{i}\left(\mathrm{p}^{\bar{U}}(\bar{v}) \rightarrow t \gg_{i} \varphi\right)\right) \quad \mathrm{PR} 2
\end{aligned}
$$

- Base case: Axiom U1. By Fig. 20, Axiom U2, and PR.

- Base case: Axiom U2. By Fig. 21, Axiom U2, and PR.

- Base case: Axiom U3. By Fig. 22, Axiom U2, and PR.

- Base case: Axiom U4. We consider three sub-cases.

1. First sub-case: $U, u \vdash(t, i, \varphi)$. Axiom $\mathrm{U} 4$ then has the form $[U, u]\left(t \gg_{i} \varphi\right) \leftrightarrow$ $\neg \mathrm{p}^{U}(u)$. The result follows by Fig. 23, Axiom U2, and PR. 


$$
\begin{aligned}
& {[\bar{U}, \bar{u}][U, u] B_{i} \varphi} \\
& \Leftrightarrow^{*}[\bar{U} \circ U,(\bar{u}, u)] B_{i} \varphi \\
& \Leftrightarrow \mathrm{p}^{\bar{U} \circ U}(\bar{u}, u) \rightarrow \bigwedge_{(\bar{u}, u) R_{i}^{\bar{U} \circ U}(\bar{v}, v)} B_{i}[\bar{U} \circ U,(\bar{v}, v)] \varphi \mathrm{U} 3 \\
& \Leftrightarrow \mathrm{p}^{\bar{U} \circ U}(\bar{u}, u) \rightarrow \bigwedge_{(\bar{u}, u) R_{i}^{\bar{U} \circ U}(\bar{v}, v)} B_{i}[\bar{U}, \bar{v}][U, v] \varphi \quad \text { U5, MR } \\
& \Leftrightarrow * \mathrm{p}^{\bar{U} \circ U}(\bar{u}, u) \rightarrow \bigwedge_{u R_{i}^{U} v} \bigwedge_{\bar{u} R_{i}^{\bar{U}} \bar{v}} B_{i}[\bar{U}, \bar{v}][U, v] \varphi \quad \text { def'n of ०, PR } \\
& \Leftrightarrow{ }^{*} \mathrm{p}^{\bar{U}}(\bar{u}) \wedge[\bar{U}, \bar{u}] \mathrm{p}^{U}(u) \rightarrow \\
& \bigwedge_{u R_{i}^{U} v} \bigwedge_{\bar{u} R_{i}^{\bar{U}} \bar{v}} B_{i}[\bar{U}, \bar{v}][U, v] \varphi \quad \text { Lem. 4.5, PR } \\
& \Leftrightarrow^{*}[\bar{U}, \bar{u}] \mathrm{p}^{U}(u) \rightarrow \\
& \bigwedge_{u R_{i}^{U} v}\left(\mathrm{p}^{\bar{U}}(\bar{u}) \rightarrow \bigwedge_{\bar{u} R_{i}^{\bar{U}} \bar{v}} B_{i}[\bar{U}, \bar{v}][U, v] \varphi\right) \quad \mathrm{PR} \\
& \Leftrightarrow{ }^{*}[\bar{U}, \bar{u}] \mathrm{p}^{U}(u) \rightarrow \bigwedge_{u R_{i}^{U} v}[\bar{U}, \bar{u}] B_{i}[U, v] \varphi \quad \text { U3, PR } \\
& \Leftrightarrow *[\bar{U}, \bar{u}] \mathrm{p}^{U}(u) \rightarrow[\bar{U}, \bar{u}] \bigwedge_{u R_{i}^{U} v} B_{i}[U, v] \varphi \quad \text { U5, PR } \\
& \Leftrightarrow{ }^{*}[\bar{U}, \bar{u}]\left(\mathrm{p}^{U}(u) \rightarrow \bigwedge_{u R_{i}^{U} v} B_{i}[U, v] \varphi\right) \quad \text { U5, PR }
\end{aligned}
$$

Fig. 22 Base case U3, proof of Lemma 4.11

$$
\begin{aligned}
& {[\bar{U}, \bar{u}][U, u] t \gg_{i} \varphi} \\
& \Leftrightarrow^{*}[\bar{U} \circ U,(\bar{u}, u)] t \gg_{i} \varphi \quad \mathrm{U} 5 \\
& \Leftrightarrow^{*} \neg \mathrm{p}^{\bar{U} \circ U}(\bar{u}, u) \quad \mathrm{U} 4,+ \\
& \Leftrightarrow{ }^{*} \neg\left(\mathrm{p}^{\bar{U}}(\bar{u}) \wedge[\bar{U}, \bar{u}] \mathrm{p}^{U}(u)\right) \text { Lem 4.5, PR } \\
& \Leftrightarrow{ }^{*}[\bar{U}, \bar{u}] \mathrm{p}^{U}(u) \rightarrow \neg \mathrm{p}^{\bar{U}}(\bar{u}) \quad \mathrm{PR} \\
& \Leftrightarrow{ }^{*}[\bar{U}, \bar{u}] \mathrm{p}^{U}(u) \rightarrow[\bar{U}, \bar{u}] \perp \quad \mathrm{U} 1, \mathrm{PR} \\
& \Leftrightarrow{ }^{*}[\bar{U}, \bar{u}] \neg \mathrm{p}^{U}(u) \quad \mathrm{U} 2
\end{aligned}
$$

Assumption of sub-case: $\quad U, u \vdash(t, i, \varphi)$

$$
\text { By (*) and Lem. 3.11: } \bar{U} \circ U,(\bar{u}, u) \vdash(t, i, \varphi)
$$

Fig. 23 Base case U4 (first sub-case), proof of Lemma 4.11

2. Second sub-case: $U, u \nvdash(t, i, \varphi)$ and $\bar{U}, \bar{u} \nvdash(t, i, \varphi)$. Axiom $\mathrm{U} 4$ then has the form $[U, u]\left(t \gg_{i} \varphi\right) \leftrightarrow\left(\mathrm{p}^{U}(u) \rightarrow t \gg_{i} \varphi\right)$. The result follows by Fig. 24, Axiom U2, and PR.

3. Third sub-case: $U, u \nvdash(t, i, \varphi)$ and $\bar{U}, \bar{u} \vdash(t, i, \varphi)$. Axiom $\mathrm{U} 4$ then has the form $[U, u]\left(t \gg_{i} \varphi\right) \leftrightarrow\left(\mathrm{p}^{U}(u) \rightarrow t \gg_{i} \varphi\right)$. The result follows by Fig. 25, Axiom U2, and PR.

- Base case: Axiom U5. By Fig. 26, Axiom U2, and PR.

- Induction case: Rule CN. Suppose $c_{k}:_{i} \varphi$ was derived by Rule CN from AX-theorem $\varphi$. Applying the definition of $c_{k}:_{i} \varphi$, it follows that $\vdash^{*} \varphi, \vdash^{*} c_{k} \gg_{i} \varphi$, and $\vdash^{*} B_{i} \varphi$. By the induction hypothesis, we have $\vdash^{*}[\bar{U}, \bar{v}] \varphi$ for arbitrary $\bar{v} \in W^{\bar{U}}$ and hence that $\vdash^{*} \bigwedge_{\bar{u} R_{i}^{\bar{U}} \bar{v}} B_{i}[\bar{U}, \bar{v}] \varphi$ by MR. But then it follows by PR that the first line of the chain of equivalences in Fig. 27 is JLCE*-derivable. The result follows by PR.

Lemma 4.12 (Update Admissibility) $\vdash^{*} \varphi$ implies $\vdash^{*}[\bar{U}, \bar{u}] \varphi$. 


$$
\begin{aligned}
& {[\bar{U}, \bar{u}][U, u] t \gg_{i} \varphi} \\
& \Leftrightarrow^{*}[\bar{U} \circ U,(\bar{u}, u)] t \gg_{i} \varphi \quad \mathrm{U} 5 \\
& \Leftrightarrow^{*} \mathrm{p}^{\bar{U} \circ U}(\bar{u}, u) \rightarrow t \gg_{i} \varphi \quad \mathrm{U} 4,++ \\
& \Leftrightarrow^{*}\left(\mathrm{p}^{\bar{U}}(\bar{u}) \wedge[\bar{U}, \bar{u}] \mathrm{p}^{U}(u)\right) \rightarrow t \gg_{i} \varphi \quad \text { Lem. 4.5, PR } \\
& \Leftrightarrow^{*}[\bar{U}, \bar{u}] \mathrm{p}^{U}(u) \rightarrow\left(\mathrm{p}^{\bar{U}}(\bar{u}) \rightarrow t \gg_{i} \varphi\right) \quad \mathrm{PR} \\
& \Leftrightarrow^{*}[\bar{U}, \bar{u}] \mathrm{p}^{U}(u) \rightarrow[\bar{U}, \bar{u}] t \gg_{i} \varphi \quad \mathrm{U} 4, * *, \mathrm{PR} \\
& \Leftrightarrow^{*}[\bar{U}, \bar{u}]\left(\mathrm{p}^{U}(u) \rightarrow t \gg_{i} \varphi\right) \quad \mathrm{U} 2
\end{aligned}
$$

Assumption of sub-case: $\quad U, u \nvdash(t, i, \varphi)$ and $\bar{U}, \bar{u} \nvdash(t, i, \varphi)$

By (**) and Lem. 3.11: $\bar{U} \circ U,(\bar{u}, u) \nvdash(t, i, \varphi)$

Fig. 24 Base case U4 (second sub-case), proof of Lemma 4.11

$$
\begin{aligned}
& {[\bar{U}, \bar{u}][U, u] t \gg_{i} \varphi} \\
& \Leftrightarrow^{*}[\bar{U} \circ U,(\bar{u}, u)] t \gg_{i} \varphi \quad \text { U5 } \\
& \Leftrightarrow^{*} \neg \mathrm{p}^{\bar{U} \circ U}(\bar{u}, u) \quad \mathrm{U} 4,+++ \\
& \Leftrightarrow^{*} \neg\left(\mathrm{p}^{\bar{U}}(\bar{u}) \wedge[\bar{U}, \bar{u}] \mathrm{p}^{U}(u)\right) \quad \text { Lem 4.5, PR } \\
& \Leftrightarrow^{*}[\bar{U}, \bar{u}] \mathrm{p}^{U}(u) \rightarrow \neg \mathrm{p}^{\bar{U}}(\bar{u}) \quad \mathrm{PR} \\
& \Leftrightarrow^{*}[\bar{U}, \bar{u}] \mathrm{p}^{U}(u) \rightarrow[\bar{U}, \bar{u}] t \gg_{i} \varphi \quad \mathrm{U} 4, * * *, \mathrm{PR} \\
& \Leftrightarrow^{*}[\bar{U}, \bar{u}]\left(\mathrm{p}^{U}(u) \rightarrow t \gg_{i} \varphi\right) \quad \mathrm{U} 2
\end{aligned}
$$

Assumption of sub-case: $\quad U, u \nvdash(t, i, \varphi)$ and $\bar{U}, \bar{u} \vdash(t, i, \varphi) \quad(* * *)$

By (***) and Lem. 3.11: $\quad \bar{U} \circ U,(\bar{u}, u) \vdash(t, i, \varphi)$

Fig. 25 Base case U4 (third sub-case), proof of Lemma 4.11

$$
\begin{aligned}
& {[\bar{U}, \bar{u}][U, u]\left[U^{\prime}, u^{\prime}\right] \varphi} \\
& \Leftrightarrow^{*}[\bar{U} \circ U,(\bar{u}, u)]\left[U^{\prime}, u^{\prime}\right] \varphi \quad \mathrm{U} 5 \\
& \Leftrightarrow^{*}\left[(\bar{U} \circ U) \circ U^{\prime},\left((\bar{u}, u), u^{\prime}\right)\right] \varphi \text { U5 } \\
& \Leftrightarrow^{*}\left[\bar{U} \circ\left(U \circ U^{\prime}\right),\left(\bar{u},\left(u, u^{\prime}\right)\right)\right] \varphi \text { Lem. } 4.6 \\
& \Leftrightarrow^{*}[\bar{U}, \bar{u}]\left[U \circ U^{\prime},\left(u, u^{\prime}\right)\right] \varphi \quad \text { U5 }
\end{aligned}
$$

Fig. 26 Base case U5, proof of Lemma 4.11

$$
\begin{array}{rlr} 
& \left(\mathrm{p}^{U} \rightarrow c_{k} \gg_{i} \varphi\right) \wedge\left(\mathrm{p}^{\bar{U}}(\bar{u}) \rightarrow \bigwedge_{\bar{u} R_{i}^{\bar{U}} \bar{v}} B_{i}[\bar{U}, \bar{v}] \varphi\right) & \\
\Leftrightarrow^{*}\left(\mathrm{p}^{U} \rightarrow c_{k} \gg_{i} \varphi\right) \wedge[\bar{U}, \bar{u}] B_{i} \varphi & \mathrm{U} 3, \mathrm{PR} \\
\Leftrightarrow^{*}[\bar{U}, \bar{u}]\left(c_{k} \gg_{i} \varphi\right) \wedge[\bar{U}, \bar{u}] B_{i} \varphi & \mathrm{U} 4, \mathrm{PR} \\
\Leftrightarrow^{*}[\bar{U}, \bar{u}] c_{k}:_{i} \varphi & \mathrm{U} 2, \mathrm{PR}
\end{array}
$$

Fig. 27 Induction case CN, proof of Lemma 4.11

Proof By induction on the length of JLCE* $^{*}$ derivations. In the base and induction cases where Rule AX was used, the result follows by Lemma 4.11. In the induction case where Rule MP was used with premises $\varphi \rightarrow \psi$ and $\varphi$, it follows by the induction hypothesis that $\vdash^{*}[\bar{U}, \bar{u}](\varphi \rightarrow \psi)$ and $\vdash^{*}[\bar{U}, \bar{u}] \varphi$. Applying Axiom U2 and 
classical propositional reasoning, it follows that $\vdash^{*}[\bar{U}, \bar{u}] \varphi \rightarrow[\bar{U}, \bar{u}] \psi$. Therefore, by Rule MP, we conclude that $\vdash^{*}[\bar{U}, \bar{u}] \psi$.

Theorem 4.13 (Admissibility) $\vdash \varphi$ if and only if $\vdash^{*} \varphi$.

Proof The left-to-right direction is shown by induction on the length of JLCE derivation and makes use of Belief Admissibility and Update Admissibility (Lemmas 4.3 and 4.12). The right-to-left direction is immediate: a JLCE* derivation is a JLCE derivation.

Theorem 4.14 (JLCE Internalization) If $\vdash \varphi$ and $i \in \mathscr{A}$, then there is a term $t \in \mathscr{T}$ such that $\vdash t:_{i} \varphi$.

Proof By JLCE* Internalization (Lemma 4.2) and Theorem 4.13.

\section{Depth and reduction}

One of the central results of many Dynamic Epistemic Logics is the Reduction Theorem, which says that every formula $\varphi$ containing an update modal $[U, u]$ can be "reduced" to a provably equivalent formula $\varphi^{\dagger}$ that does not contain any update modals. The Reduction Theorem typically plays an important role in the proof of completeness, and this is so for the theories JLCE and JLCE* as well. However, in these theories, Reduction has the following variant form: each formula $\varphi$ can be "reduced" to a provably equivalent formula $\varphi^{\dagger}$ whose update modals occur only within the scope of a term, by which we mean that update modals appear only in subformulas $\psi$ that themselves occur within a subformula having the form $t \gg_{i} \psi$. As we will see later, this version of Reduction is sufficient for proving completeness for JLCE and JLCE*.

Since we saw in Theorem 4.13 that JLCE and JLCE* derive the same theorems, it suffices for us to prove Reduction for JLCE*. As in Dynamic Epistemic Logic van Ditmarsch et al. (2007), we proceed by defining a notion of "depth" for the language; this notion is sometimes called "complexity" by other authors.

$$
\begin{array}{ll}
d(q) & :=1, \text { for } q \in \mathscr{P} \cup\{\perp\} \\
d(\varphi \rightarrow \psi) & :=1+\max \{d(\varphi), d(\psi)\} \\
d\left(B_{i} \varphi\right) & :=1+d(\varphi) \\
d\left(t \gg_{i} \varphi\right) & :=2+\max \{d(t), d(\varphi)\} \\
d([U, u] \varphi) & :=(4+d(U))^{4+d(\varphi)} \cdot d(\varphi) \\
d(U) & :=\left|W^{U}\right|+\max _{u \in W^{U}} d\left(\mathrm{p}^{U}(u)\right) \\
d\left(c_{k}\right) & :=1 \\
d\left(x_{k}\right) & :=1 \\
d(t \cdot{ } s) & :=1+\max \{d(t), d(s), d(\varphi)\} \\
d(t+s) & :=1+\max \{d(t), d(s)\} \\
d(! t) & :=1+d(t)
\end{array}
$$

Fig. 28 Definition of a function $d: \mathscr{F} \cup \mathscr{T} \rightarrow \mathbb{N}$. Note this definition is adapted from van Ditmarsch et al. 2007 


$$
\begin{array}{ll}
q^{\dagger} & =q \text { if } q \in \mathscr{P} \cup\{\perp\} \\
(\varphi \rightarrow \psi)^{\dagger} & =\varphi^{\dagger} \rightarrow \psi^{\dagger} \\
\left(B_{i} \varphi\right)^{\dagger} & =B_{i}\left(\varphi^{\dagger}\right) \\
\left(t \gg_{i} \varphi\right)^{\dagger} & =t \gg_{i} \varphi \\
([U, u] q)^{\dagger} & =\left(\mathrm{p}^{U}(u)\right)^{\dagger} \rightarrow q \text { if } q \in \mathscr{P} \cup\{\perp\} \\
([U, u](\varphi \rightarrow \psi))^{\dagger} & =([U, u] \varphi)^{\dagger} \rightarrow([U, u] \psi)^{\dagger} \\
\left([U, u] B_{i} \varphi\right)^{\dagger} & =\left(\mathrm{p}^{U}(u)\right)^{\dagger} \rightarrow \bigwedge_{u R_{i}^{U}} B_{i}([U, v] \varphi)^{\dagger} \\
\left([U, u]\left(t \gg_{i} \varphi\right)\right)^{\dagger} & = \begin{cases}\neg\left(\mathrm{p}^{U}(u)\right)^{\dagger} & \text { if } U, u \vdash(t, i, \varphi) \\
\left(\mathrm{p}^{U}(u)\right)^{\dagger} \rightarrow t \gg_{i} \varphi & \text { if } U, u \nvdash(t, i, \varphi)\end{cases} \\
\left([U, u]\left[U^{\prime}, u^{\prime}\right] \varphi\right)^{\dagger} & =\left(\left[U \circ U^{\prime},\left(u, u^{\prime}\right)\right] \varphi\right)^{\dagger}
\end{array}
$$

Fig. 29 Definition of a function $\dagger: \mathscr{F} \rightarrow \mathscr{F}$, Theorem 5.2

Definition 5.1 (Depth) The equations in Fig. 28 define a function $d: \mathscr{F} \cup \mathscr{T} \rightarrow \mathbb{N}$ that maps each $o \in \mathscr{F} \cup \mathscr{T}$ to a natural number $d(o)$ called the depth of $o$. For each $n \in \mathbb{N}^{+}$, we define the sets

$$
\begin{aligned}
& \mathscr{F}^{(n)}:=\{\varphi \in \mathscr{F} \mid d(\varphi) \leq n\} \\
& \mathscr{T}^{(n)}:=\left\{t \in \mathscr{T} \mid t \text { occurs in some } \varphi \in \mathscr{F}^{(n)}\right\} \\
& \mathscr{U}^{(n)}:=\mathscr{U}\left(\mathscr{T}^{(n)}, \mathscr{F}^{(n)}\right)
\end{aligned}
$$

and define the language $\mathrm{UL}^{(n)}:=\left(\mathscr{T}^{(n)}, \mathscr{F}^{(n)}\right)$.

With the definition of depth in hand, we now define how to "reduce" a given formula to a JLCE* -equivalent formula whose update modals occur only within the scope of a term.

Theorem 5.2 (Reduction) The schematic equations in Fig. 29 define a function $\dagger$ : $\mathscr{F} \rightarrow \mathscr{F}$ that maps each formula $\varphi$ to a formula $\varphi^{\dagger}$ such that $\varphi \Leftrightarrow^{*} \varphi^{\dagger}$. Further, the schematic equations in Fig. 29 are depth-respecting: for each $\mathscr{F}$-instance of a schematic equation in Fig. 29, the function $\dagger$ is applied on the left-hand side to a formula whose depth is strictly greater than that of any formula on the right-hand side to which $\dagger$ is applied.

Proof To show that equations in Fig. 29 define a function that takes each formula $\varphi$ to a formula $\varphi^{\dagger}$, we argue by induction on $n \in \mathbb{N}^{+}$that the equations in Fig. 29 define a function $\uparrow_{n}: \mathscr{F}^{(n)} \rightarrow \mathscr{F}^{(n)}$. The equations defining $\uparrow_{n}$ are obtained from those in Fig. 29 as follows: the $\dagger$ on the left-hand side of an equation is to be replaced by $\uparrow_{n}$, each $\dagger$ on the right-hand side of an equation is to be replaced by $\uparrow_{n-1}$, and any equation that then contains $\dagger_{0}$ on its right-hand side is to be omitted. It is easy to see that $\dagger_{1}$ is well-defined, and it is similarly easy to argue that $\dagger_{n+1}$ is well-defined if $\dagger_{n}$ is well-defined. The latter argument requires us to prove that our equations in Fig. 29 are depth-respecting, which is a dull exercise in unravelling definitions and reasoning with inequalities. To get a flavor for these arguments, see Fig. 30. Arguments for some of the other inequalities can be found by adapting Figs. $4(2-4,6)$ from Renne $(2008 \mathrm{a})$; 


$$
\begin{aligned}
& d\left(\left[U \circ U^{\prime},\left(u, u^{\prime}\right)\right] \varphi\right) \\
= & \left(4+d\left(U \circ U^{\prime}\right)\right)^{4+d(\varphi)} \cdot d(\varphi) \\
= & \left(4+\left|W^{U}\right| \cdot\left|W^{U^{\prime}}\right|+\max _{\left(v, v^{\prime}\right) \in W^{U \circ U^{\prime}}}\left\{d\left(\neg[U, v] \neg \mathrm{p}^{U^{\prime}}\left(v^{\prime}\right)\right)\right\}\right)^{4+d(\varphi)} \cdot d(\varphi) \\
= & \left(5+\left|W^{U}\right| \cdot\left|W^{U^{\prime}}\right|+\right. \\
& \left.\max _{v^{\prime} \in W^{U^{\prime}}}\left\{(4+d(U))^{5+d\left(\mathrm{p}^{U^{\prime}}\left(v^{\prime}\right)\right)} \cdot\left(1+d\left(\mathrm{p}^{U^{\prime}}\left(v^{\prime}\right)\right)\right)\right\}\right)^{4+d(\varphi)} \cdot d(\varphi) \\
\leq & \left(5+\left|W^{U}\right| \cdot\left|W^{U^{\prime}}\right|+(4+d(U))^{4+d\left(U^{\prime}\right)} \cdot d\left(U^{\prime}\right)\right)^{4+d(\varphi)} \cdot d(\varphi) \\
< & \left(2 \cdot(4+d(U))^{4+d\left(U^{\prime}\right)} \cdot d\left(U^{\prime}\right)\right)^{4+d(\varphi)} \cdot d(\varphi) \\
< & \left((4+d(U))^{5+d\left(U^{\prime}\right)} \cdot d\left(U^{\prime}\right)\right)^{4+d(\varphi)} \cdot d(\varphi) \\
< & (4+d(U))^{\left(5+d\left(U^{\prime}\right)\right) \cdot(4+d(\varphi))} \cdot\left(4+d\left(U^{\prime}\right)\right)^{4+d(\varphi)} \cdot d(\varphi) \\
= & (4+d(U))^{20+5 \cdot d(\varphi)+4 \cdot d\left(U^{\prime}\right)+d\left(U^{\prime}\right) \cdot d(\varphi)} \cdot\left(4+d\left(U^{\prime}\right)\right)^{4+d(\varphi)} \cdot d(\varphi) \\
< & (4+d(U))^{4+\left(4+d\left(U^{\prime}\right)\right)^{5} \cdot d(\varphi)} \cdot\left(4+d\left(U^{\prime}\right)\right)^{4+d(\varphi)} \cdot d(\varphi) \\
\leq & (4+d(U))^{4+\left(4+d\left(U^{\prime}\right)\right)^{4+d(\varphi)} \cdot d(\varphi)} \cdot\left(4+d\left(U^{\prime}\right)\right)^{4+d(\varphi)} \cdot d(\varphi) \\
= & d\left([U, u]\left[U^{\prime}, u^{\prime}\right] \varphi\right)
\end{aligned}
$$

Fig. 30 Proof that $d\left(\left[U \circ U^{\prime},\left(u, u^{\prime}\right)\right] \varphi\right)<d\left([U, u]\left[U^{\prime}, u^{\prime}\right] \varphi\right)$

arguments for the remaining inequalities are not too difficult to obtain. We therefore have by induction on $n \in \mathbb{N}^{+}$that $\dagger_{n}$ is well-defined for each $n \in \mathbb{N}^{+}$. Next, we define a function $\dagger$ mapping formulas to formulas by setting $\varphi^{\dagger}:=\varphi^{\dagger} d(\varphi)$ and then argue that $\dagger$ is the unique function satisfying the equations in Fig. 29. ${ }^{10}$

All that remains is for us to argue by induction on formula depth that $\varphi \Leftrightarrow \Leftrightarrow^{*} \varphi^{\dagger}$. Almost all of the cases are straightforward adaptations of the standard arguments in Dynamic Epistemic Logic van Ditmarsch et al. (2007), with the exception of the following cases that we handle in detail.

- Case $B_{i} \psi$.

Since $\dagger$ is depth-respecting, we have $d(\psi)<d\left(B_{i} \psi\right)$ and hence that $\psi \Leftrightarrow \Leftrightarrow^{*} \psi^{\dagger}$ by the induction hypothesis. Thus $B_{i} \psi \Leftrightarrow \Leftrightarrow^{*} B_{i} \psi^{\dagger}$ by modal reasoning (using Lemma 4.3 instead of Rule BN). Since $B_{i} \psi^{\dagger}=\left(B_{i} \psi\right)^{\dagger}$, it follows that $B_{i} \psi \Leftrightarrow \Leftrightarrow^{*}$ $\left(B_{i} \psi\right)^{\dagger}$.

- Case $[U, u] B_{i} \psi$.

Let $S:=\left\{v \in W^{U} \mid u R_{i}^{U} v\right\}$. If $S=\emptyset$, then a conjunction $\bigwedge_{u R_{i}^{U} v} \chi_{v}$ ranging over $S$ is equal to $T$. It therefore follows by Axiom U3 and classical propositional reasoning that $[U, u] B_{i} \psi \Leftrightarrow^{*} \top$, and it follows by the definition of $\dagger$ and classical propositional reasoning that $\left([U, u] B_{i} \psi\right)^{\dagger} \Leftrightarrow^{*} \top$. We therefore have that $[U, u] B_{i} \psi \Leftrightarrow \Leftrightarrow^{*}\left([U, u] B_{i} \psi\right)^{\dagger}$, as desired.

\footnotetext{
${ }^{10}$ In a bit more detail, making frequent use of the fact that the equations in Fig. 29 are depth-respecting, we proceed in the following way. First, we argue by induction on $n \in \mathbb{N}^{+}$that for each $\varphi \in \mathscr{F}^{(n)}$ and each $k \in \mathbb{N}$, we have $\varphi^{\dagger} d(\varphi)=\varphi^{\dagger} d(\varphi)+k$. Using this, we argue that the function $\dagger$ defined by $\varphi^{\dagger}:=\varphi^{\dagger} d(\varphi)$ satisfies the equations in Fig. 29. Finally, we argue by induction on $n \in \mathbb{N}^{+}$that if $r: \mathscr{F} \rightarrow \mathscr{F}$ is another function satisfying the equations in Fig. 29, then $\varphi^{r}=\varphi^{\dagger}$ for each $\varphi \in \mathscr{F}^{(n)}$.
} 
So suppose $S \neq \emptyset$ and hence that there is a $v \in S$. Since $\dagger$ is depth-respecting, we have $d\left(\mathrm{p}^{U}(u)\right)<d\left([U, u] B_{i} \psi\right)$ and $d([U, v] \psi)<d\left([U, u] B_{i} \psi\right)$ and hence that $\mathrm{p}^{U}(u) \Leftrightarrow \Leftrightarrow^{*}\left(\mathrm{p}^{U}(u)\right)^{\dagger}$ and $[U, v] \psi \Leftrightarrow \Leftrightarrow^{*}([U, v] \psi)^{\dagger}$ by the induction hypothesis. Applying modal reasoning (using Lemma 4.3 instead of Rule BN), we have $\mathrm{p}^{U}(u) \Leftrightarrow^{*}\left(\mathrm{p}^{U}(u)\right)^{\dagger}$ and $B_{i}[U, v] \psi \Leftrightarrow^{*} B_{i}([U, v] \psi)^{\dagger}$. Since $v \in S$ was chosen arbitrarily, it follows by Axiom U3, the definition of $\dagger$, and classical propositional reasoning that $[U, u] B_{i} \psi \Leftrightarrow \Leftrightarrow^{*}\left([U, u] B_{i} \psi\right)^{\dagger}$, as desired.

- Case $[U, u]\left(t \gg_{i} \psi\right)$.

Since $\dagger$ is depth-respecting, we have $d\left(\mathrm{p}^{U}(u)\right)<d\left([U, u]\left(t \gg_{i} \psi\right)\right)$ and hence that $\mathrm{p}^{U}(u) \Leftrightarrow * \quad\left(\mathrm{p}^{U}(u)\right)^{\dagger}$ by the induction hypothesis. Applying classical propositional reasoning and Axiom U4, it follows that $[U, u]\left(t \gg_{i} \psi\right) \Leftrightarrow{ }^{*}$ $\left([U, u]\left(t \gg_{i} \psi\right)\right)^{\dagger}$.

We have therefore shown that every formula $\varphi$ can be reduced to a JLCE* -equivalent formula $\varphi^{\dagger}$.

\section{Semantics}

The semantics of UL is our adaptation of a Kripke-style semantics for Justification Logic due to Fitting (2005) and Mkrtychev (1997).

Definition 6.1 To say that $M$ is a Fitting model means that $M$ is a tuple $\left(W^{M}, R^{M}\right.$, $V^{M}, A^{M}$ ) whose components satisfy the following.

- $W^{M}$ is a nonempty set whose members are called worlds (in $M$ ).

- $R^{U}: \mathscr{A} \rightarrow \wp\left(W^{U} \times W^{U}\right)$ assigns a transitive binary relation $R_{i}^{U} \subseteq W^{U} \times W^{U}$ on $W^{U}$ to each agent $i \in \mathscr{A}$.

- $V^{U}: \mathscr{P} \rightarrow \wp\left(W^{U}\right)$ assigns a set $V^{U}(p)$ of worlds in $M$ to each propositional letter $p \in \mathscr{P}$.

- $A^{U}: \mathscr{A} \rightarrow\left(\mathscr{T} \times \mathscr{F} \rightarrow \wp\left(W^{U}\right)\right)$ assigns a set $A_{i}(t, \varphi)$ of worlds in $M$ to each term-agent-formula triple $(t, i, \varphi) \in \mathscr{T} \times \mathscr{A} \times \mathscr{F}$ subject to the following schematic conditions that together make a function of type $\mathscr{A} \rightarrow(\mathscr{T} \times \mathscr{F} \rightarrow$ $\left.\wp\left(W^{U}\right)\right)$ an evidence function.

Constant Specification: $c \in \mathscr{C}$ and $\mathrm{AX} \vdash \varphi$ imply $A_{i}^{M}(c, \varphi)=W^{M}$.

Application: $A_{i}^{M}(t, \varphi \rightarrow \psi) \cap A_{i}^{M}(s, \varphi) \subseteq A_{i}^{M}(t \cdot \varphi s, \psi)$.

Sum: $A_{i}^{M}(t, \varphi) \cup A_{i}^{M}(s, \varphi) \subseteq A_{i}^{M}(t+s, \varphi)$.

Checker: $A_{i}^{M}(t, \varphi) \subseteq A_{i}^{M}\left(! t, t:_{i} \varphi\right)$.

Monotonicity: $\Gamma R_{i}^{M} \Delta$ and $\Gamma \in A_{i}^{M}(t, \varphi)$ imply $\Delta \in A_{i}^{M}(t, \varphi)$.

A pointed Fitting model is a pair $(M, \Gamma)$ consisting of a Fitting model $M$ and a world $\Gamma \in W^{U} ; \Gamma$ is said to be the point of $(M, \Gamma)$.

Definition 6.2 (Truth) Given a pointed Fitting model $(M, \Gamma)$ and a formula $\varphi \in \mathscr{F}$, we write $M, \Gamma \models \varphi$ to mean that $\varphi$ is true at $(M, \Gamma)$; the negation of $M, \Gamma \models \varphi$ is written $M, \Gamma \not \models \varphi$. We define when it is that a formula is true at a pointed Fitting model according to the following induction on formula depth. 
- $\quad M, \Gamma \models p_{k}$ means that $\Gamma \in V^{M}\left(p_{k}\right)$.

- $M, \Gamma \not \models \perp$.

- $M, \Gamma \models \varphi_{1} \rightarrow \varphi_{2}$ means that $M, \Gamma \not \varphi_{1}$ or $M, \Gamma \models \varphi_{2}$.

- $M, \Gamma \models B_{i} \varphi$ means that $M, \Delta \models \varphi$ for each $\Delta \in W^{M}$ with $\Gamma R_{i}^{M} \Delta$.

- $\quad M, \Gamma \models t \gg_{i} \varphi$ means that $\Gamma \in A_{i}^{M}(t, \varphi)$.

- $\quad M, \Gamma \models[U, u] \varphi$ means that either $M, \Gamma \not \models \mathrm{p}^{U}(u)$ or else both $M, \Gamma \models \mathrm{p}^{U}(u)$ and $M[U],(\Gamma, u) \models \varphi$, where the components of the tuple $M[U]$ are defined as follows.

$$
\begin{aligned}
W^{M[U]} & :=\left\{(\Delta, v) \in W^{M} \times W^{U} \mid M, \Delta \models \mathrm{p}^{U}(v)\right\} \\
R_{i}^{M[U]} & :=\left\{\left((\Delta, v),\left(\Delta^{\prime}, v^{\prime}\right)\right) \mid \Delta R_{i}^{M} \Delta^{\prime} \& v R_{i}^{U} v^{\prime}\right\} \\
A_{i}^{M[U]}(t, \psi) & :=\left\{(\Delta, v) \mid \Delta \in A_{i}^{M}(t, \psi) \& U, v \nvdash(t, i, \psi)\right\} \\
V^{M[U]}\left(p_{k}\right) & :=\left\{(\Delta, v) \mid \Delta \in V^{M}\left(p_{k}\right)\right\}
\end{aligned}
$$

To say that $\varphi \in \mathscr{F}$ is valid, written $\models \varphi$, means that we have $M, \Gamma \models \varphi$ for each pointed Fitting model $(M, \Gamma)$.

Lemma 6.3 (Update Correctness) Let $(M, \Gamma)$ be a pointed Fitting model and $(U, u)$ be a pointed update frame. If $M, \Gamma \models \mathrm{p}^{U}(u)$, then $M[U]$ is a Fitting model.

Proof $W^{M[U]}$ is nonempty because $M, \Gamma \models \mathrm{p}^{U}(u) . R_{i}^{M[U]}$ is transitive because each of $R_{i}^{M}$ and $R_{i}^{U}$ is transitive. To prove that $A^{M[U]}$ is an evidence function, we check each of the defining properties in turn.

- Constant Specification: $c \in \mathscr{C}$ and AX $\vdash \varphi$ imply $A_{i}^{M[U]}(c, \varphi)=W^{M[U]}$.

Suppose $c \in \mathscr{C}$ and $\mathrm{AX} \vdash \varphi$. Choose an arbitrary $(\Delta, v) \in W^{M[U]}$. We have that $\Delta \in A_{i}^{M}(c, \varphi)$ because $A^{M}$ is an evidence function. Further, we have $U, v \nvdash$ $(c, i, \varphi)$ (see Fig. 1). Hence $(\Delta, v) \in A_{i}^{M[U]}\left(c_{k}, \varphi\right)$ by the definition of $A_{i}^{M[U]}$.

- Application: $A_{i}^{M[U]}(t, \varphi \rightarrow \psi) \cap A_{i}^{M[U]}(s, \varphi) \subseteq A_{i}^{M[U]}(t \cdot \varphi s, \psi)$.

Suppose $(\Delta, v) \in A_{i}^{M[U]}(t, \varphi \rightarrow \psi) \cap A_{i}^{M[U]}(s, \varphi)$. It follows by the definition of $A_{i}^{M[U]}$ that $\Delta \in A_{i}^{M}(t, \varphi \rightarrow \psi) \cap A_{i}^{M}(s, \varphi), U, v \nvdash(t, i, \varphi \rightarrow \psi)$, and $U, v \nvdash(s, i, \varphi)$. The first of these items implies $\Delta \in A_{i}^{M}(t \cdot \varphi s)$ because $A^{M}$ is an evidence function. The second two of the three items together imply that $U, v \nvdash\left(t \cdot{ }_{\varphi} s, i, \psi\right)$ (see Fig. 1). But the latter and $\Delta \in A_{i}^{M}\left(t \cdot{ }_{\varphi} s\right)$ together imply that $(\Delta, v) \in A_{i}^{M[U]}(t \cdot \varphi s, \psi)$ by the definition of $A_{i}^{M[U]}$.

- Sum: $A_{i}^{M[U]}(t, \varphi) \cup A_{i}^{M[U]}(s, \varphi) \subseteq A_{i}^{M[U]}(t+s, \varphi)$. Similar to the argument for Application.

- Checker: $A_{i}^{M[U]}(t, \varphi) \subseteq A_{i}^{M[U]}\left(! t, t:_{i} \varphi\right)$. Similar to the argument for Application.

- Monotonicity: $(\Delta, v) R_{i}^{M[U]}\left(\Delta^{\prime}, v^{\prime}\right)$ and $(\Delta, v) \in A_{i}^{M[U]}(t, \varphi)$ imply $\left(\Delta^{\prime}, v^{\prime}\right) \in$ $A_{i}^{M[U]}(t, \varphi)$.

Suppose $(\Delta, v) \in A_{i}^{M[U]}(t, \varphi)$ and $(\Delta, v) R_{i}^{M[U]}\left(\Delta^{\prime}, v^{\prime}\right)$. It follows that $\Delta \in$ $A_{i}^{M}(t, \varphi)$ and $U, v \nvdash(t, i, \varphi)$ by the definition of $A_{i}^{M[U]}$ and that $\Delta R_{i}^{M} \Delta^{\prime}$ and $v R_{i}^{U} v^{\prime}$ by the definition of $R_{i}^{M[U]}$. Since $A^{M}$ is an evidence function, it follows 
that $\Delta^{\prime} \in A_{i}(t, \varphi)$. Since $U, v \nvdash(t, i, \varphi)$ and $v R_{i}^{U} v^{\prime}$, it follows by Lemma 3.7 that $U, v^{\prime} \nvdash(t, i, \varphi)$. But the latter and $\Delta^{\prime} \in A_{i}^{M}(t, \varphi)$ together imply that $\left(\Delta^{\prime}, v^{\prime}\right) \in$ $A_{i}^{M[U]}(t, \varphi)$ by the definition of $A_{i}^{M[U]}$.

In the definition of truth (Definition 6.2), the cases for formulas having the form $[U, u] \varphi$ delegate part of their work to the $U$-calculus (Fig. 1). This may seem strange because we are admitting the $U$-calculus-a syntactic notion-into our semantics. However, it is our intention for evidence eliminations to respect the intended meanings of the term-forming operations (described earlier in the Sect. 3). This ensures that the elimination of one or more parts of a combination $t$ of multiple pieces of evidence shall affect $t$ itself. Therefore, some simple theory describing the logical consequences that the elimination of a simple term has on more complex terms is a necessary part of the semantics. One may also take some comfort in the fact that the $U$-calculus is a simple, decidable theory.

Definition 6.4 (Isomorphism) To say that $f$ is an isomorphism between Fitting models $M$ and $M^{\prime}$ means that $f$ is a function of type $W^{M} \rightarrow W^{M^{\prime}}$ satisfying each of the following: $f$ is a bijection, $\Gamma R_{i}^{M} \Delta$ if and only if $f(\Gamma) R_{i}^{M^{\prime}} f(\Delta)$ for each $(\Gamma, \Delta) \in W^{M} \times W^{M}$ and each $i \in \mathscr{A}, \Gamma \in V^{M}(p)$ if and only if $f(\Gamma) \in V^{M^{\prime}}(p)$ for each $\Gamma \in W^{M}$ and each $p \in \mathscr{P}$, and $\Gamma \in A_{i}^{M}(t, \psi)$ if and only if $f(\Gamma) \in A_{i}^{M^{\prime}}(t, \psi)$ for each $\Gamma \in W^{M}$ and each $(t, i, \psi) \in \mathscr{T} \times \mathscr{A} \times \mathscr{F}$.

Theorem 6.5 (Isomorphism Equivalence) For each isomorphism $f$ between Fitting models $M$ and $M^{\prime}$, each formula $\varphi \in \mathscr{F}$, and each pointed update frame $(U, u) \in \mathscr{U}$ such that there exists an $\Omega \in W^{M}$ satisfying $M, \Omega \models \mathrm{p}^{U}(u)$ and $M^{\prime}, f(\Omega) \models \mathrm{p}^{U}(u)$, we have each of the following items.

1. $M, \Gamma \models \varphi$ if and only if $M^{\prime}, f(\Gamma) \models \varphi$ for each $\Gamma \in W^{M}$.

2. $f^{U}: W^{M[U]} \rightarrow W^{M^{\prime}[U]}$ defined by setting $f^{U}(\Delta, v):=(f(\Delta), v)$ is an isomorphism between $M[U]$ and $M^{\prime}[U]$.

Proof By induction on $n:=\max \{d(U), d(\varphi)\} \in \mathbb{N}^{+}$. The base case $n=1$ is vacuously true because $d(U) \geq 2$. So we proceed to the induction case. Item 6.5 is proved by considering the possible syntactic forms that $\varphi$ might have. First, if $\varphi$ has one of the forms $q \in \mathscr{P} \cup\{\perp\}, \psi \rightarrow \chi$, or $B_{i} \psi$, then the argument is just as in modal logic (Blackburn et al. 2001). So let us consider the remaining forms that $\varphi \in \mathscr{F}$ might have.

- Case: $\varphi$ has the form $t \gg_{i} \psi$.

$M, \Gamma \models t \gg_{i} \psi$ means that $\Gamma \in A_{i}^{M}(t, \psi)$. Since $f$ is an isomorphism between $M$ and $M^{\prime}$, the latter holds if and only if $f(\Gamma) \in A_{i}^{M^{\prime}}(t, \psi)$. But this is what it means to have $M^{\prime}, f(\Gamma) \models t \gg_{i} \psi$.

- Case: $\varphi$ has the form $\left[U^{\prime}, u^{\prime}\right] \psi$.

Since $d\left(\mathrm{p}^{U^{\prime}}\left(u^{\prime}\right)\right)<d\left(\left[U^{\prime}, u^{\prime}\right] \psi\right)$, it follows by the induction hypothesis that $M, \Gamma \models \mathrm{p}^{U^{\prime}}\left(u^{\prime}\right)$ if and only if $M^{\prime}, f(\Gamma) \models \mathrm{p}^{U^{\prime}}\left(u^{\prime}\right)$. Further, under the assumption that both $M, \Gamma \models \mathrm{p}^{U^{\prime}}\left(u^{\prime}\right)$ and $M^{\prime}, f(\Gamma) \models \mathrm{p}^{U^{\prime}}\left(u^{\prime}\right)$, since $d\left(U^{\prime}\right)<$ $d\left(\left[U^{\prime}, u^{\prime}\right] \psi\right)$, it follows by the induction hypothesis that $f^{U^{\prime}}$ is an isomorphism between $M\left[U^{\prime}\right]$ and $M^{\prime}\left[U^{\prime}\right]$ and hence that $M\left[U^{\prime}\right],\left(\Gamma, u^{\prime}\right) \models \psi$ if and only if 
$M^{\prime}\left[U^{\prime}\right],\left(f(\Gamma), u^{\prime}\right) \models \psi$. Applying the definition of truth (Definition 6.2) and the definition of $f^{U^{\prime}}$, we have proved that $M, \Gamma \models\left[U^{\prime}, u^{\prime}\right] \psi$ if and only if $M^{\prime}, f(\Gamma) \models\left[U^{\prime}, u^{\prime}\right] \psi$.

This completes the proof of Item 6.5. To prove Item 6.5, we prove that $f^{U}$ is an isomorphism between $M[U]$ and $M^{\prime}[U]$.

- $f^{U}$ is a bijection.

First, we argue that $f^{U}$ is surjective. Proceeding, $\left(\Delta^{\prime}, v\right) \in W^{M^{\prime}[U]}$ means that $M^{\prime}, \Delta^{\prime} \models \mathrm{p}^{U}(v)$. Since $f$ is surjective, there is a $\Delta \in W^{M}$ such that $f(\Delta)=\Delta^{\prime}$. Further, since $d\left(\mathrm{p}^{U}(v)\right)<d(U)$, it follows by the induction hypothesis that $M^{\prime}, f(\Delta) \models \mathrm{p}^{U}(v)$ if and only if $M, \Delta \models \mathrm{p}^{U}(v)$. But the latter is what it means to have $(\Delta, v) \in W^{M[U]}$. Since $f^{U}(\Delta, v)=(f(\Delta), v)=\left(\Delta^{\prime}, v\right)$, we have shown that $f^{U}$ is surjective.

We now argue that $f^{U}$ is injective. Proceeding, assume that

$$
f^{U}\left(\Delta_{1}, v_{1}\right)=f^{U}\left(\Delta_{2}, v_{2}\right) .
$$

It follows by the definition of $f^{U}$ that $f\left(\Delta_{1}\right)=f\left(\Delta_{2}\right)$ and $v_{1}=v_{2}$. Since $f$ is injective, it follows that $\Delta_{1}=\Delta_{2}$ and $v_{1}=v_{2}$. So $f^{U}$ is injective.

- $\quad(\Delta, v) R_{i}^{M[U]}\left(\Delta^{\prime}, v^{\prime}\right)$ if and only if $f^{U}(\Delta, v) R_{i}^{M^{\prime}[U]} f^{U}\left(\Delta^{\prime}, v\right)$.

$(\Delta, v) R_{i}^{M[U]}\left(\Delta^{\prime}, v^{\prime}\right)$ means that $\Delta R_{i}^{M} \Delta^{\prime}$ and $v R_{i}^{U} v^{\prime}$. Since $f$ is an isomorphism, the latter is equivalent to $f(\Delta) R_{i}^{M^{\prime}} f\left(\Delta^{\prime}\right)$ and $v R_{i}^{U} v^{\prime}$, which is what it means to have $f^{U}(\Delta, v) R_{i}^{M^{\prime}[U]} f^{U}\left(\Delta^{\prime}, v\right)$.

- $\quad(\Delta, v) \in V^{M[U]}\left(p_{k}\right)$ if and only if $f^{U}(\Delta, v) \in V^{M^{\prime}[U]}\left(p_{k}\right)$.

$(\Delta, v) \in V^{M[U]}\left(p_{k}\right)$ means that $\Delta \in V^{M}\left(p_{k}\right)$. Since $f$ is an isomorphism, the latter is equivalent to $f(\Delta) \in V^{M^{\prime}}\left(p_{k}\right)$. But this is what it means to have $f^{U}(\Delta, v) \in V^{M^{\prime}[U]}\left(p_{k}\right)$.

- $\quad(\Delta, v) \in A_{i}^{M[U]}(t, \psi)$ if and only if $f^{U}(\Delta, v) \in A_{i}^{M^{\prime}[U]}(t, \psi)$.

$(\Delta, v) \in A_{i}^{M[U]}(t, \psi)$ means that $\Delta \in A_{i}^{M}(t, \psi)$ and $U, v \nvdash(t, i, \psi)$. Since $f$ is an isomorphism, the latter conjunction is equivalent to the statement that $f(\Delta) \in$ $A_{i}^{M^{\prime}}(t, \psi)$ and $U, v \nvdash(t, i, \psi)$. But this is what it means to have $f^{U}(\Delta, v) \in$ $A_{i}^{M^{\prime}[U]}(t, \psi)$.

Theorem 6.6 (Soundness) $\vdash \varphi$ implies $\models \varphi$ for each $\varphi \in \mathscr{F}$.

Proof By Admissibility (Theorem 4.13), it suffices for us to prove that $\vdash^{*} \varphi$ implies $\models$ $\varphi$. Proceeding, we first prove that $A X \vdash \chi$ implies $\models \chi$ by induction on the length of $A X$ derivations. In the base case, we must check each of the AX-axioms. Most of the arguments for these axioms are straightforward, making use of standard arguments in Dynamic Epistemic Logic (van Ditmarsch et al. 2007) or the fact that the function $A^{M}$ in a Fitting model $M$ satisfies the defining properties of an evidence function. Perhaps the trickiest Axiom is U5, so we shall handle this axiom in detail.

- Axiom U5 is sound; that is, $\models[U, u]\left[U^{\prime}, u^{\prime}\right] \varphi \leftrightarrow\left[U \circ U^{\prime},\left(u, u^{\prime}\right)\right] \varphi$.

While much of this argument is standard in Dynamic Epistemic Logic (van Ditmarsch et al. 2007), the Justification Logic-specific aspects of AX introduce some complications, so we shall handle this argument in full. Let $(M, \Gamma)$ 
be a pointed Fitting model. We may assume without loss of generality that $M, \Gamma \models \neg[U, u] \neg \mathrm{p}^{U^{\prime}}\left(u^{\prime}\right)$ (for the result otherwise follows easily by the definition of truth, Definition 6.2). Proceeding, it suffices by Isomorphism Equivalence (Theorem 6.5) and the definition of truth (Definition 6.2) for us to prove that the function $f: W^{M[U]\left[U^{\prime}\right]} \rightarrow W^{M\left[U \circ U^{\prime}\right]}$ defined by setting $f\left((\Delta, v), v^{\prime}\right):=\left(\Delta,\left(v, v^{\prime}\right)\right)$ is an isomorphism between $M[U]\left[U^{\prime}\right]$ and $M\left[U \circ U^{\prime}\right]$.

- $f$ is a bijection.

$\left((\Delta, v), v^{\prime}\right) \in W^{M[U]\left[U^{\prime}\right]}$ if and only if $M, \Delta \models \neg[U, v] \neg \mathrm{p}^{U^{\prime}}\left(v^{\prime}\right)$. But the latter is equivalent to the statement that $\left(\Delta,\left(v, v^{\prime}\right)\right) \in W^{M\left[U \circ U^{\prime}\right]}$.

- $\Omega_{1} R_{i}^{M[U]\left[U^{\prime}\right]} \Omega_{2}$ if and only if $f\left(\Omega_{1}\right) R_{i}^{M\left[U \circ U^{\prime}\right]} f\left(\Omega_{2}\right)$.

We have $\left((\Delta, v), v^{\prime}\right) R_{i}^{M[U]\left[U^{\prime}\right]}\left((\Omega, w), w^{\prime}\right)$ if and only if $\Delta R_{i}^{M} \Omega, v R_{i}^{U} w$, and $v^{\prime} R_{i}^{U^{\prime}} w^{\prime}$. But the latter trio is equivalent to the statement that $\left(\Delta,\left(v, v^{\prime}\right)\right)$ $R_{i}^{M\left[U \circ U^{\prime}\right]}\left(\Omega,\left(w, w^{\prime}\right)\right)$.

- $\Omega \in V^{M[U]\left[U^{\prime}\right]}\left(p_{k}\right)$ if and only if $f(\Omega) \in V^{M\left[U \circ U^{\prime}\right]}\left(p_{k}\right)$. $\left((\Delta, v), v^{\prime}\right) \in V^{M[U]\left[U^{\prime}\right]}\left(p_{k}\right)$ and $\left(\Delta,\left(v, v^{\prime}\right)\right) \in V^{M\left[U \circ U^{\prime}\right]}\left(p_{k}\right)$ are each equivalent to the statement that $\Delta \in V^{M}\left(p_{k}\right)$.

- $\Omega \in A_{i}^{M[U]\left[U^{\prime}\right]}(t, \psi)$ if and only if $f(\Omega) \in A_{i}^{M\left[U \circ U^{\prime}\right]}(t, \psi)$.

$\left((\Delta, v), v^{\prime}\right) \in A_{i}^{M[U]\left[U^{\prime}\right]}(t, \psi)$ means that $\Delta \in A_{i}^{M}(t, \psi), U, v \nvdash(t, i, \psi)$, and $U^{\prime}, v^{\prime} \nvdash(t, i, \psi)$. Applying Composition (Lemma 3.11), we have $\Delta \in$ $A_{i}(t, \psi)$ and $\left(U \circ U^{\prime}\right),\left(v, v^{\prime}\right) \nvdash(t, i, \psi)$. But this is what it means to have $\left(\Delta,\left(v, v^{\prime}\right)\right) \in A_{i}^{M\left[U \circ U^{\prime}\right]}(t, \psi)$.

Conclusion: Axiom U5 is valid.

We have argued that each $\mathrm{AX}$-axiom is valid. For the induction case, assume we derived $\mathrm{AX} \vdash c_{k}:_{i} \varphi$ by Rule $\mathrm{CN}$ from $\mathrm{AX}$-theorem $\varphi$. By the induction hypothesis, $\models \varphi$. Letting $(M, \Gamma)$ be a pointed Fitting model, $\models \varphi$ implies $M, \Delta \models \varphi$ for each $\Delta \in W^{M}$ satisfying $\Gamma R_{i}^{M} \Delta$. Further, since $\varphi$ is an AX-theorem, it follows that $\Gamma \in A_{i}^{M}\left(c_{k}, \varphi\right)$ because $A^{M}$ is an evidence function. Therefore $M, \Gamma \models c_{k}:_{i} \varphi$. Since $(M, \Gamma)$ was chosen arbitrarily, we have shown that $\models c_{k}:_{i} \varphi$. Conclusion: $\mathrm{AX} \vdash \varphi$ implies $=\varphi$. By the standard argument for the validity of Rule MP (Blackburn et al. 2001), it therefore follows that $\vdash^{*} \varphi$ implies $\models \varphi$.

Theorem 6.7 (Consistency) JLCE is consistent; that is, $\nvdash \perp$.

Proof Define the Fitting model $M$ by setting $W^{M}:=\{\Gamma\}, R_{i}^{M}:=\{(\Gamma, \Gamma)\}$ for each $i \in \mathscr{A}, V^{M}(p):=\emptyset$ for each $p \in \mathscr{P}$, and $A_{i}^{M}(t, \varphi)=W^{M}$ for each $(t, i, \varphi) \in$ $\mathscr{T} \times \mathscr{A} \times \mathscr{F}$. (Note that $M$ is indeed a Fitting model: $W^{M}$ is nonempty, $R_{i}^{M}$ is transitive for each $i \in \mathscr{A}$, and $A^{M}$ is an evidence function.) Since $M, \Gamma \not \models \perp$, it follows by Soundness (Theorem 6.6) that $\nvdash \perp$.

Theorem 6.8 (Completeness) $\models \varphi$ implies $\vdash \varphi$ for each $\varphi \in \mathscr{F}$.

Proof Since JLCE* ${ }^{*}$ is a subsystem of JLCE, it suffices for us to argue that $\models \varphi$ implies $\vdash^{*} \varphi$. Our proof proceeds by a canonical model argument, so let us first make some preliminary definitions. Let $S$ be a set of formulas. If $S$ is finite, then we define 


$$
\bigwedge S:= \begin{cases}\top & \text { if } S=\emptyset \\ \bigwedge_{\psi \in S} \psi & \text { otherwise. }\end{cases}
$$

To say that $S$ is inconsistent means that there is a finite subset $S^{\prime} \subseteq S$ such that $\vdash^{*}\left(\bigwedge S^{\prime}\right) \rightarrow \perp$. To say that $S$ is consistent means that $S$ is not inconsistent. To say that $S$ is maximal consistent means that $S$ is consistent and adding any formula not already present in $S$ will result in a set that is inconsistent. By a Lindenbaum Argument, any consistent set of formulas may be extended to a maximal consistent set of formulas. The canonical model is the structure $M^{*}:=\left(W^{*}, R^{*}, V^{*}, A^{*}\right)$ whose components are defined as follows.

- $\quad W^{*}$ is the set of all maximal consistent sets of formulas.

- $R^{*}: \mathscr{A} \rightarrow \wp\left(W^{*} \times W^{*}\right)$ is defined by

$$
R_{i}^{*}:=\left\{(\Gamma, \Delta) \in W^{*} \times W^{*} \mid \forall \varphi \in \mathscr{F}: B_{i} \varphi \in \Gamma \text { implies } \varphi \in \Delta\right\} .
$$

$-\quad A^{*}: \mathscr{A} \rightarrow\left(\mathscr{T} \times \mathscr{F} \rightarrow \wp\left(W^{*}\right)\right)$ is defined by setting

$$
A_{i}^{*}(t, \varphi):=\left\{\Gamma \in W^{*} \mid\left(t \gg_{i} \varphi\right) \in \Gamma\right\} .
$$

- $\quad V^{*}: \mathscr{P} \rightarrow \wp\left(W^{*}\right)$ is defined by setting $V(p):=\left\{\Gamma \in W^{*} \mid p \in \Gamma\right\}$.

To see that $M^{*}$ is in fact a Fitting model: $W^{*}$ is nonempty because the set $\Gamma$ of all formulas true at the pointed Fitting model $(M, \Gamma)$ from the proof of Theorem 6.7 is maximal consistent; for each $i \in \mathscr{A}$, the relation $R_{i}^{*}$ is transitive by the standard argument in modal logic Blackburn et al. (2001); $A^{*}$ is an evidence function by Rule AX, classical propositional reasoning, and Axioms E1-E4.

The key property of $M^{*}$ that we now wish to prove is the Truth Lemma: for each $\varphi \in \mathscr{F}$ and each $\Gamma \in W^{*}$, we have that $\varphi \in \Gamma$ if and only if $M^{*}, \Gamma \models \varphi$. The proof is by induction on formula depth.

- Cases $q \in \mathscr{P} \cup\{\perp\}, \varphi \rightarrow \psi$, and $B_{i} \varphi$ follow by the standard arguments in modal logic (Blackburn et al. 2001).

- Case $t \gg_{i} \varphi$.

By the definition of $A^{*}$, we have $\left(t \gg_{i} \varphi\right) \in \Gamma$ if and only if $\Gamma \in A_{i}^{*}(t, \varphi)$. But the latter is what it means to have $M^{*}, \Gamma \models t \gg_{i} \varphi$.

- Case $[U, u] \varphi$.

$[U, u] \varphi \in \Gamma$ is equivalent to $([U, u] \varphi)^{\dagger} \in \Gamma$ by Reduction (Theorem 5.2) and the maximal consistency of $\Gamma$. By the induction hypothesis, we have $([U, u] \varphi)^{\dagger} \in \Gamma$ if and only if $M^{*}, \Gamma \models([U, u] \varphi)^{\dagger}$. Applying Soundness (Theorem 6.6), Reduction (Theorem 5.2), and the definition of truth (Definition 6.2), it follows $M^{*}, \Gamma \models$ $([U, u] \varphi)^{\dagger}$ is equivalent to $M^{*}, \Gamma \models[U, u] \varphi$.

This completes the proof of the Truth Lemma. The completeness argument is then easy: suppose $\nvdash^{*} \chi$. It follows that $\{\neg \chi\}$ is consistent and so may be extended to a maximal consistent set $\Gamma \in W^{M^{*}}$. Since $\neg \chi \in \Gamma$, it follows by the Truth Lemma that $M^{*}, \Gamma \models \neg \chi$ and thus that $M^{*}, \Gamma \not \models \chi$ by the definition of truth (Definition 6.2). Since $M^{*}$ is a Fitting model, we have shown that $\nvdash^{*} \chi$ implies $\not \models \chi$. It follows that $\models \chi$ implies $\vdash^{*} \chi$. 
We conclude this section with a theorem that provides an important connection between JLCE and the $U$-calculus. The theorem tells us that whenever $\vdash t \gg_{i} \varphi$, it is not possible to eliminate agent $i$ 's evidence $t$ relevant to $\varphi$. (See our discussion in Remark 3.6.)

Theorem 6.9 (Non-Elimination) $\vdash t \gg_{i} \varphi$ implies $U, u \nvdash(t, i, \varphi)$.

Proof It follows by Soundness (Theorem 6.6) that $\vdash t \gg_{i} \varphi$ implies $\models t \gg_{i} \varphi$. Let $M$ be the Fitting model from the proof of Consistency (Theorem 6.7). Suppose toward a contradiction that we have $U, u \vdash(t, i, \varphi)$ for some $(U, u) \in \mathscr{U}$. Since the derivability of $U, u \vdash(t, i, \varphi)$ does not depend on the value of $\mathrm{p}^{U}(u)$, we could then assume without loss of generality that $\mathrm{p}^{U}(u)=\top$. Applying the definition of truth (Definition 6.2), we would then have that $\Gamma \notin A_{i}^{M[U]}(t, \varphi)$ and hence that $M[U],(\Gamma, u) \not \models t \gg_{i} \varphi$, contradicting the fact that $\models t \gg_{i} \varphi$.

\section{Formalized example}

We now use JLCE to formalize our email example from the beginning of this paper. Since we are interested only in the evidence and beliefs of Bob $(\mathfrak{B})$ and Charlie $(\mathfrak{C})$, we define our set $\mathscr{A}$ of agents by $\mathscr{A}:=\{\mathfrak{B}, \mathfrak{C}\}$. We let $O$ ("open") abbreviate propositional letter $p_{0}$ and $C$ ("cheese") abbreviate propositional letter $p_{1}$, and we write $O \rightarrow C$ ("open implies cheese") to describe Anne's plan to bring the cheese if the store is open. Email messages $x_{1}$ through $x_{4}$ provide us with our initial setup $X$, a conjunction of the formulas

- $x_{1}:_{\mathfrak{B}}(O \rightarrow C)$ (" $x_{1}$ is Bob's evidence that $O \rightarrow C$ "),

- $x_{1}: \mathfrak{C}(O \rightarrow C)$ (" $x_{1}$ is Charlie's evidence that $O \rightarrow C$ "),

- $x_{2}: \mathfrak{B}\left(x_{1}: \mathfrak{C}(O \rightarrow C)\right)$ (" $x_{2}$ is Bob's evidence that $x_{1}: \mathfrak{C}(O \rightarrow C)$ "),

- $x_{3}::_{\mathfrak{B}} O$ (" $x_{3}$ is Bob's evidence that $O$ "),

- $x_{3}: \mathfrak{C} O$ (" $x_{3}$ is Charlie's evidence that $O$ "), and

- $x_{4}: \mathfrak{B}_{\mathfrak{B}}\left(x_{3}: \mathfrak{C} O\right)$ (" $x_{4}$ is Bob's evidence that $x_{3}: \mathfrak{C} O$ ").

It follows by classical propositional reasoning and Lemma 4.1 that $\vdash X \rightarrow\left(x_{1} \cdot O\right.$ $\left.x_{3}\right):_{\mathfrak{B}} C$ and $\vdash X \rightarrow\left(x_{1} \cdot o x_{3}\right): \mathfrak{C} C$; that is, given our setup $X$, each of Bob and Charlie has evidence $x_{1} \cdot o x_{3}$ that Anne is bringing the cheese.

Let us now look at Bob's evidence about Charlie's evidence. First, it follows by Lemma 4.1 and Internalization (Theorem 4.14) that there is a term $t$ such that

$$
\vdash t: \mathfrak{B}\left(x_{1}: \mathfrak{C}(O \rightarrow C) \rightarrow\left(x_{3}: \mathfrak{C} O \rightarrow\left(x_{1} \cdot o x_{3}\right): \mathfrak{C} C\right)\right)
$$

Defining the term $s$ by setting

$$
s:=\left(t \cdot\left(x_{1}: \mathfrak{C}(O \rightarrow C)\right) x_{2}\right) \cdot\left(x_{3}: \mathfrak{c} O\right) x_{4},
$$

it follows by Lemma 4.1 and classical propositional reasoning that

$$
\vdash X \rightarrow s: \mathfrak{B}\left(x_{1} \cdot o x_{3}\right): \mathfrak{C} C
$$




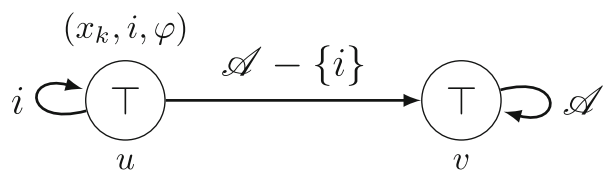

Fig. 31 The update frame $\mathrm{PRI}_{i}^{(k, \varphi)}$

that is, "given setup $X$, Bob has evidence $s$ that Charlie has evidence $x_{1} \cdot o x_{3}$ that $C$." So from the initial situation $X$ given by messages $x_{1}$ through $x_{4}$, each of Bob and Charlie has evidence $x_{1} \cdot O x_{3}$ that Anne is bringing the cheese, and Bob has evidence $s$ that Charlie has evidence $x_{1} \cdot o x_{3}$ that Anne is bringing the cheese.

Now let us examine how Anne's private email $x_{5}$ to Bob affects Bob's evidence. Adapting the Dynamic Epistemic Logic definition of the private announcement to an agent (described in the introduction; see also: Baltag and Moss (2004); Renne (2008b); van Ditmarsch et al. (2007)), we define the update frame $\mathrm{PRI}{ }_{i}^{(k, \varphi)}$, called the private elimination of agent $i$ 's evidence $x_{k}$ relevant to $\varphi$, as follows.

$$
\begin{aligned}
W^{\mathrm{PRI}_{i}^{(k, \varphi)}} & :=\{u, v\} & \mathrm{p}^{\mathrm{PRI}}{ }_{i}^{(k, \varphi)}(w):=\top \text { for } w \in\{u, v\} \\
R_{i}^{\mathrm{PRI}_{i}^{(k, \varphi)}} & :=\{(u, u),(v, v)\} & \mathrm{V}^{\mathrm{PRI}}{ }_{i}^{(k, \varphi)}(u):=\left\{\left(x_{k}, i, \varphi\right)\right\} \\
R_{j}^{\mathrm{PRI}_{i}^{(k, \varphi)}} & :=\{(u, v),(v, v)\} \text { if } j \neq i & \mathrm{v}^{\mathrm{PRI}}{ }_{i}^{(k, \varphi)}(v):=\emptyset
\end{aligned}
$$

We picture $\mathrm{PRI}_{i}^{(k, \varphi)}$ in Fig. 31. We will use the update $\left(\mathrm{PRI}_{\mathfrak{B}}^{(3, O)}, u\right)$ to represent the effect of Anne's final message $x_{5}$. Using PR to denote the use of classical propositional reasoning, we then have the following.

1. $\mathrm{PRI}_{\mathfrak{B}}^{(3, O)}, u \vdash\left(x_{3}, \mathfrak{B}, O\right)$

By Axiom EV of the $\mathrm{PRI}_{\mathfrak{B}}^{(3, O)}$-calculus (Fig. 1).

2. $\mathrm{PRI}_{\mathfrak{B}}^{(3, O)}, u \vdash\left(x_{1} \cdot o x_{3}, \mathfrak{B}, C\right)$

By line 1 and Rule EAR of the $\mathrm{PRI}_{\mathfrak{B}}^{(3, O)}$-calculus (Fig. 1).

3. $\vdash\left[\mathrm{PRI}_{\mathfrak{B}}^{(3, O)}, u\right]\left(\left(x_{1} \cdot o x_{3}\right) \gg_{\mathfrak{B}} C\right) \rightarrow \perp$

By line 2, Axiom U4 (with $\mathrm{p}^{\mathrm{PRI}} \mathrm{B}_{\mathfrak{B}}^{(3,0)}(u)=\top$ ), and PR.

4. $\left[\mathrm{PRI} \mathbf{B}_{\mathfrak{B}}^{(3, O)}, u\right]\left(\left(x_{1} \cdot o x_{3}\right) \gg_{\mathfrak{B}} C\right) \rightarrow\left[\mathrm{PRI}_{\mathfrak{B}}^{(3,0)}, u\right] \perp$

By line 3, Axiom U1 (with $\mathrm{p}^{\mathrm{PRI}} \mathrm{I}_{\mathfrak{B}}^{(3,0)}(u)=\top$ ), and PR.

5. $\vdash\left[\mathrm{PRI}_{\mathfrak{B}}^{(3, O)}, u\right] \neg\left(\left(x_{1} \cdot o x_{3}\right) \gg_{\mathfrak{B}} C\right)$

By line 4, Axiom U2, and PR.

6. $\vdash\left[\mathrm{PRI}_{\mathfrak{B}}^{(3, O)}, u\right] \neg\left(\left(x_{1} \cdot o x_{3}\right) \gg_{\mathfrak{B}} C\right) \rightarrow\left[\mathrm{PRI}_{\mathfrak{B}}^{(3, O)}, u\right] \neg\left(\left(x_{1} \cdot o x_{3}\right):_{\mathfrak{B}} C\right)$

By Def. 3.4, PR, Rule UN, and Axiom U2.

7. $\vdash\left[\mathrm{PRI}_{\mathfrak{B}}^{(3, O)}, u\right] \neg\left(\left(x_{1} \cdot o x_{3}\right):_{\mathfrak{B}} C\right)$

By lines 5 and 6 and Rule MP.

This is, after Anne's private message to Bob eliminating Bob's evidence $x_{3}$ relevant to $O$, it is not the case that $x_{1} \cdot o x_{3}$ is Bob's evidence that $C$ (Anne brings the cheese). And yet we have the following.

8. $\mathrm{PRI}_{\mathfrak{B}}^{(3, O)}, u \nvdash\left(t, \mathfrak{B}, x_{1}: \mathfrak{C}(O \rightarrow C) \rightarrow\left(x_{3}: \mathfrak{C} O \rightarrow\left(x_{1} \cdot o x_{3}\right): \mathfrak{C} C\right)\right)$ 
By (5), Def. 3.4, PR, and Non-Elimination (Theorem 6.9).

9. $\mathrm{PRI}_{\mathfrak{B}}^{(3, O)}, u \nvdash\left(x_{2}, \mathfrak{B}, x_{1}: \mathfrak{C}(O \rightarrow C)\right)$

Inspection of the $\mathrm{PRI}_{\mathfrak{B}}^{(3,0)}$-calculus (Fig. 1).

10. $\mathrm{PRI}_{\mathfrak{B}}^{(3, O)}, u \nvdash\left(x_{4}, \mathfrak{B}, x_{\mathfrak{3}}: \mathfrak{C} O\right)$

Inspection of the $\mathrm{PRI}_{\mathfrak{B}}^{(3,0)}$-calculus (Fig. 1).

11. $\mathrm{PRI}_{\mathfrak{B}}^{(3, O)}, u \nvdash\left(s, \mathfrak{B},\left(x_{1} \cdot o x_{3}\right): \mathfrak{C} C\right)$

By lines 8,9 , and 10 and inspection of the $\mathrm{PRI}_{\mathfrak{B}}^{(3,0)}$-calculus.

12. $\vdash X \rightarrow s \gg_{\mathfrak{B}}\left(x_{1} \cdot o x_{3}\right): \mathfrak{C} C$

By (6), Def. 3.4, and PR.

13. $\vdash X \rightarrow\left[\mathrm{PRI}_{\mathfrak{B}}^{(3, O)}, u\right] s \gg_{\mathfrak{B}}\left(x_{1} \cdot o x_{3}\right): \mathfrak{C} C$

By lines 11 and 12, Axiom U4 (with $\mathrm{p}^{\mathrm{PRI}_{\mathfrak{B}}^{(3, O)}, u}(u)=\top$ ), and PR.

14. $\vdash X \rightarrow B_{\mathfrak{B}}\left(x_{1} \cdot o x_{3}\right): \mathfrak{C} C$

By (6), Def. 3.4, and PR.

15. $\vdash X \rightarrow\left[\mathrm{PRI}_{\mathfrak{B}}^{(3, O)}, u\right] B_{\mathfrak{B}}\left(x_{1} \cdot o x_{3}\right): \mathfrak{C} C$

By line 14, Axiom U3 (with $\mathrm{p}^{\mathrm{PRI}}{ }_{\mathfrak{B}}^{(3, O)}, u(u)=\top$ and $\left.R_{\mathfrak{B}}^{\mathrm{PRI}_{\mathfrak{B}}^{(3, O)}}(u)=\{(u, u)\}\right)$, and PR.

16. $\vdash X \rightarrow\left[\mathrm{PRI}_{\mathfrak{B}}^{(3, O)}, u\right] s:_{\mathfrak{B}}\left(x_{1} \cdot o x_{3}\right): \mathfrak{C} C$

By lines 13 and 15, Def. 3.4, Axiom U2, and PR.

In words: given setup $X$, after Anne's private message to Bob eliminating Bob's evidence $x_{3}$ relevant to $O$, it is (still) the case that Bob has evidence $s$ that Charlie has evidence $x_{1} \cdot o x_{3}$ that Anne will bring the cheese (line 16), despite the fact that Bob himself does not consider $x_{1} \cdot o x_{3}$ evidence that Anne will bring the cheese (line 7).

\section{Conclusion}

The work in this paper is part of a larger project, Dynamic Justification Logic, whose aim is to combine the frameworks of Dynamic Epistemic Logic and Justification Logic in order to reason about belief and evidence dynamics arising from multi-agent communications. The role of this paper in the project is to introduce multi-agent evidence elimination, one kind of dynamic operation on multi-agent evidence that causes an agent to set aside a piece of evidence relevant to a given assertion and then determine how this affects other pieces of evidence as a result of the ways in which evidence may be logically combined in Justification Logic. In earlier work (Renne 2008a), the author studied a notion of evidence introduction, whereby a piece of evidence may be introduced as relevant for a given assertion; however, the particular operation the author defined there lacks the tight integration with the semantics of Dynamic Epistemic Logic used for evidence elimination in this paper, and so the techniques developed here might well be adapted to a notion of multi-agent evidence introduction as well.

Yavorskaya (2008) studied the first multi-agent languages for Justification Logic. Yavorskaya's multi-agent languages are based on a variation of the basic fragment of UL. The variation may be obtained from the basic fragment of UL by making certain changes to the term-formation grammar in $\mathscr{G}_{0}$, including, among other changes, 
the creation of a superscript-labeled copy $t^{i}$ of each term $t$ for each agent $i \in \mathscr{A}$ and the requirement that a colon-formula $t:_{i} \varphi$ is well-formed if and only if $t$ is a term $s^{i}$ labeled by the superscript of agent $i$. Yavorskaya's work investigates certain "interactions" between the terms of different agents, in the sense of acceptance of principles such as $t^{i}:_{i} \varphi \rightarrow\left(!_{i}^{j} t^{i}\right)^{j}:_{j}\left(t^{i}:_{i} \varphi\right)$ ("agent $j$ can check agent $i$ 's evidence") or $t^{i}:_{i} \varphi \rightarrow\left(\uparrow_{i}^{j} t^{i}\right)^{j}:_{j} \varphi$ ("agent $j$ trusts agent $i$ 's evidence"). Such interactions are essentially static because the Yavorskaya languages lack update models, and hence the interaction principles endorsed by a given model for a Yavorskaya-language remain fixed once and for all. By way of contrast, update modals allow UL to describe transitions between models that bring about changes in the agents' evidence and beliefs, which is an essentially dynamic notion. So it would be natural to investigate "dynamic" versions of Yavorskaya's languages and principles, though this is something we leave for future work. Further, the exact connection between languages with Yavorskayastyle terms (where each agent has his or her own disjoint set of terms) and languages with UL-style terms (where the agents share a single set of terms) is not yet well understood. In particular, a natural question for future research is the following "multi-agent realization" question: given a sub-theory $T$ of our theory JLCE such that $T$ is in the basic fragment of UL, is there a theory $T^{\prime}$ in an appropriate Yavorskaya-style language such that every $T$-theorem can be converted into (or "realized as") a $T^{\prime}$-theorem by adding agent superscripts to terms and, conversely, every $T^{\prime}$-theorem can be converted into a $T$-theorem by omitting all agent superscripts? The author conjectures that this "multi-agent realization" question has an affirmative answer, though investigation of this question is left for future work.

Beyond the operations of evidence elimination and introduction, there are a number of natural directions in which one might proceed, including the introduction of preferences and preference change over evidence, which would allow us to tie an agent's beliefs (or the relative strength among his or her various beliefs) to his or her preference ordering on evidence. This work would naturally dovetail and complement recent work in Dynamic Epistemic Logic on Belief Revision and preference upgrade (Baltag and Smets 2007; van Benthem 2004; van Benthem and Liu 2007). It might also be interesting to compare the semantic topologic-based approach to evidence recently proposed by van Benthem and Pacuit (2011) to the present Justification Logic-style syntactic notion of evidence. Indeed, there notions similar to evidence elimination (and introduction) are studied for a less syntactical point of view.

Finally, our work has natural connections with the leaner public announcementbased Justification Logic system of evidence introduction due to Bucheli et al. (2010). The latter work is focused on finding the Justification Logic analog of Plaza's modal logic of public announcements. The key in this work is to take the agent's evidence $t$ about the truth of a statement $\psi$ after the hypothetical future announcement of $\varphi$ as sufficient for the agent having $t$ as evidence for $\psi$ after $\varphi$ is in fact announced. Thus the key evidence introduction schema (in multi-agent format) is

$$
t:_{i}(\varphi \rightarrow[\varphi] \psi) \rightarrow[\varphi] t:_{i} \psi
$$


In a follow-up paper by Bucheli et al. (2011), this approach is varied by having a certain piece of evidence $\Uparrow t$ that is derived from $t$ as support for $\psi$ after the announcement, leading to the evidence introduction schema (again in multi-agent format)

$$
t:_{i}(\varphi \rightarrow[\varphi] \psi) \rightarrow[\varphi] \Uparrow t:_{i} \psi
$$

The latter is more in the spirit of (a static variation of) the generalized step-by-step inference-based reasoning studied by Velázquez-Quesada (2009), which itself suggests a number of interesting new dynamic operations on evidence for future study.

Acknowledgements The author expresses his deep gratitude to Roman Kuznets for providing in-depth consultation on the proof of JLCE Internalization in addition to a number of other useful comments and suggestions, all of which greatly improved the text. The author also wishes to thank the first referee for extensive and thorough reports that were extremely helpful in the preparation of the final version of the text. The author is also grateful for the second referee's feedback, which improved global readability and organization and corrected some localized omissions as well. Finally, the author thanks Rineke Verbrugge and Barteld Kooi for their helpful comments that also led to improvements of the text.

Open Access This article is distributed under the terms of the Creative Commons Attribution Noncommercial License which permits any noncommercial use, distribution, and reproduction in any medium, provided the original author(s) and source are credited.

\section{References}

Artemov, S. (2001). Explicit provability and constructive semantics. The Bulletin of Symbolic Logic, 7(1), $1-36$.

Artemov, S. (2008). The logic of justification. The Review of Symbolic Logic, 1(4), 477-513.

Artemov, S., \& Nogina, E. (2005). Introducing justification into epistemic logic. Journal of Logic and Computation, 15(6), 1059-1073.

Baltag, A., \& Moss, L. S. (2004). Logics for epistemic programs. Synthese, 139(2), 165-224.

Baltag, A., Moss, L. S., \& Solecki, S. (1998). The logic of common knowledge, public announcements, and private suspicions. In: I. Gilboa (Ed.), Proceedings of the 7th conference on theoretical aspects of rationality and knowledge (TARK VII), Evanston, IL, USA. (pp. 43-56).

Baltag A., \& Smets S. (2007). A qualitative theory of dynamic interactive belief revision. In G. Bonanno, W. van der Hoek, \& M. Wooldridge (Eds.), Selected papers from LOFT'06.

Baltag, A., van Ditmarsch, H. P., \& Moss, L. S. (2008). Epistemic logic and information update. In P. Adriaans \& J. van Benthem (Eds.), Handbook on the philosophy of information (pp. 369-463). Dordrecht: Elsevier.

Blackburn, P., de Rijke, M., \& Venema, Y. (2001). Modal logic. Cambridge: Cambridge University Press.

Bucheli, S., Kuznets, R., Sack, J., \& Studer, T. (2010). Justified belief change. In X. Arrazola, \& M. Ponte (Eds.), Proceedings of the second ILCLI international workshop on logic and philosophy of knowledge, communication, and action (LogKCA-10).

Bucheli, S., Kuznets, R., Studer, T. (2011). Partial realization in dynamic justification logic. In L. Beklemishev, \& R. de Queiroz (Eds.), Logic, language, information and computation (Vol. 6642, pp. 35-51). Lecture notes in computer science. Berlin, Heidelberg: Springer.

Fitting, M. (2005). The logic of proofs, semantically. Annals of Pure and Applied Logic, 132(1), 1-25. Fitting, M. (2009). Reasoning with justifications. In D. Makinson, J. Malinowski, \& H. Wansing (Eds.), Towards mathematical philosophy, vol. 28 of trends in logic (pp. 107-123). Netherlands: Springer.

Gerbrandy, J. (1999). Bisimulations on Planet Kripke. Ph.D. thesis, Institute for Logic, Language, and Computation, University of Amsterdam. ILLC Dissertation Series DS-1999-01.

Gerbrandy, J., \& Groeneveld, W. (1997). Reasoning about information change. Journal of Logic, Language and Information, 6(2), 147-169. 
Kuznets, R. (2008). Complexity issues in justification logic. Ph.D. thesis, CUNY Ph.D. Program in Computer Science.

Mkrtychev, A. (1997). Models for the logic of proofs. In S. Adian \& A. Nerode (Eds.), Logical foundations of computer science (Vol. 1234, pp. 266-275). Lecture notes in computer science.

Plaza, J. (1989). Logics of public communications. In Z. W. Ras (Ed.), Proceedings of the fourth international symposium on methodologies for intelligent systems (ISMIS 1989), North-Holland.

Plaza, J. (2007). Logics of public communications. Synthese 158(2), 165-179. Reprint of Plaza (1989).

Renne, B. (2006). Public and private communication are different: Results on relative expressivity. Synthese, 165(2), 225-245.

Renne, B. (2008a). Dynamic Epistemic Logic with justification. Ph.D. thesis, The City University of New York.

Renne, B. (2008b). A survey of dynamic epistemic logic. Manuscript.

Renne, B. (2011) Simple evidence elimination in justification logic. In P. Girard, O. Roy, \& M. Marion (Eds.), Dynamic formal epistemology. (Vol. 351, Chap. 7, pp. 127-149). Synthese library. Berlin: Springer.

van Benthem, J. (2004). Dynamic logic for belief revision. Journal of Applied Non-Classical Logics, 14(2), 129-155.

van Benthem, J. (2006). One is a lonely number: Logic and communication. In: Z. Chatzidakis, P. Koepke, \& W. Pohlers (Eds.), Logic colloquium '02 (Vol. 27). Lecture notes in logic. Association for Symbolic Logic.

van Benthem, J., \& Liu, F. (2007). Dynamic logic of preference updgrade. Journal of Applied Non-Classical Logics, 17(2), 157-182.

van Benthem, J., \& Pacuit, E. (2011). Dynamic logics of evidence-based belief. Manuscript.

van Benthem, J., van Eijck, J., \& Kooi, B. (2006). Logics of communication and change. Information and Computation, 204(11), 1620-1662.

van Ditmarsch, H., van der Hoek, W., \& Kooi, B. (2007). Dynamic Epistemic Logic. Berlin: Springer.

Velázquez-Quesada, F. R. (2009). Inference and update. Synthese, 169, 283-300.

Yavorskaya (Sidon), T. (2008). Interacting explicit evidence systems. Theory of Computing Systems, 43, 272-293. 